\title{
Hyperpolarization Methods for MRS
}

Boyd M. Goodson, Nicholas Whiting, Aaron M. Coffey, Panayiotis Nikolaou, Fan Shi,

Brogan M. Gust, Max E. Gemeinhardt, Roman V. Shchepin, Jason G. Skinner, Jonathan R.

Birchall, Michael J. Barlow, Eduard Y. Chekmenev*

Boyd M. Goodson

Southern Illinois University, Department of Chemistry and Biochemistry

Carbondale, IL, 62901, United States

Nicholas Whiting

The University of Texas MD Anderson Cancer Center, Department of Cancer Systems

Imaging

Houston, TX, 77030, United States

Aaron M. Coffey

Vanderbilt University, Institute of Imaging Science (VUIIS), Department of Radiology

Nashville, TN, 37232, United States

Panayiotis Nikolaou

Vanderbilt University, Institute of Imaging Science (VUIIS), Department of Radiology

Nashville, TN, 37232, United States

Fan Shi

Southern Illinois University, Department of Chemistry and Biochemistry 
Carbondale, IL, 62901, United States

Brogan M. Gust

Southern Illinois University, Department of Chemistry and Biochemistry

Carbondale, IL, 62901, United States

Max E. Gemeinhardt

Southern Illinois University, Department of Chemistry and Biochemistry

Carbondale, IL, 62901, United States

Roman V. Shchepin

Vanderbilt University, Institute of Imaging Science (VUIIS), Department of Radiology

Nashville, TN, 37232, United States

Jason G. Skinner

University of Nottingham, School of Medicine

Nottingham, NG7 2UH, UK

Jonathan R. Birchall

University of Nottingham, School of Medicine

Nottingham, NG7 2UH, UK

Michael J. Barlow

University of Nottingham, School of Medicine

Nottingham, NG7 2UH, UK 


\section{Eduard Y. Chekmenev*}

Vanderbilt University, Institute of Imaging Science (VUIIS), Department of Radiology, Department of Biomedical Engineering, Department of Biochemistry, Vanderbilt-Ingram Cancer Center (VICC)

Nashville, TN, 37232, United States

$116121^{\text {st }}$ Ave South

MCN AA-1105

Nashville, TN 37232, United States

E-mail: eduard.chekmenev@vanderbilt.edu

Phone: 615-322-1329

Fax: 615-322-0734

Short Biographies:

Boyd Goodson graduated from Princeton University in 1995 and earned his PhD in chemistry in 1999 with Alexander Pines at U.C. Berkeley. Following postdoctoral work with Ahmed Zewail at Caltech, in 2002 Goodson joined the faculty at Southern Illinois University Carbondale and was promoted to full professor in 2014. Goodson's research interests concern NMR/MRI sensitivity enhancement and hyperpolarization.

Nicholas Whiting, b1983. PhD (supervisor Prof. Boyd Goodson) 2010, Southern Illinois University, Carbondale, IL, USA. NSF International Research Fellow at the University of Nottingham, UK (with Dr. Michael Barlow and Prof. Peter Morris). Odyssey and NCI Cancer Prevention Fellow (with Prof. Pratip Bhattacharya) at UT MD Anderson Cancer Center. Aaron M. Coffey, PhD 2014, Vanderbilt University, Nashville, TN, USA. Postdoctoral Fellow at Vanderbilt University Institute of Imaging Science (with Prof. Eduard Y. Chekmenev). Co-authored $>30$ peer-reviewed articles covering advanced MR detection 
hardware and utilizing hyperpolarization techniques to enable MR contrast agents for in vivo molecular imaging for improved human health.

Panayiotis Nikolaou, b1978, PhD (supervisor Prof. Boyd Goodson) 2010, Southern Illinois University, Carbondale, IL, USA. Currently: Post-Doctoral Fellow, Vanderbilt University, Nashville, TN USA (with Dr. Eduard Chekmenev) at the Institute of Imaging Science. Fan Shi. After graduating with BS/MS degrees from Zhengzhou University (China), he joined the Goodson Lab at SIUC (USA) in 2011. His research has concerned the development and application of SABRE catalysts - particularly under heterogeneous conditions ("HET-SABRE"). Fan graduated with a $\mathrm{PhD}$ in 2015, and is now an intern with Cabot Labs in Chicago, IL.

Brogan Gust graduated with a B.S. degree in chemistry from Southern Illinois University Carbondale in 2013. While at SIUC, he was under the guidance of Boyd Goodson researching NMR enhancement using Xenon-129 hyperpolarization. Max Gemeinhardt joined the Goodson Lab at SIUC in 2011 as undergraduate working on pH-sensing SPIONs. He graduated in 2012 with a B.S. degree and is currently pursuing a M.S. degree at SIUC in the Goodson Lab, where his current research interests include cryptophane host-guest inclusion complexes and SABRE NMR enhancement. Roman V. Shchepin, b1978, PhD (Prof. Patrick Dussault) 2006 in University of NebraskaLincoln (Lincoln, NE). Postdoctoral Fellow at Vanderbilt University Department of Chemistry (2007-2009) with Prof. Ned A. Porter and VUIIS (2010-2013) with Prof. Eduard Y. Chekmenev. He is currently Research Instructor at VUIIS with main focus on the chemistry of hyperpolarized NMR/MRI. Coauthored $>30$ peer-reviewed original research publications.

Jason G. Skinner. b1989. M.Phys., 2012. PhD research student (supervisors: Prof. I. Hall, Prof. P. Morris, Dr. M. Barlow), University of Nottingham, UK. Research interests include 
spin-exchange optical pumping, hyperpolarised noble gas lung MRI, and disease detection via exhaled volatile organic compounds.

Jonathan R. Birchall, b1991, M.Phys., 2013, PhD research student (supervisors: Prof. I. Hall, Dr. M. Barlow, Prof. J. Owers-Bradley), University of Nottingham, UK. Research interests include development and analysis of Spin-Exchange Optical Pumping techniques for functional human lung imaging.

Michael J. Barlow, b1958, PhD (Supervisor Prof. Brian Ridley FRS) 1988 University of Essex, UK. Postdoctoral Fellow at MOD Malvern, Durham University, Surrey University, Imperial College London and Rutherford Appleton Lab, Oxford. Instrument Manager - MAG team NASA Cassini Mission. Faculty Fellow University of Nottingham. Co-authored $>20$ peer-reviewed publications in the areas of semiconductor physics, space physics, solid state NMR, laser physics and hyperpolarized MRI.

Eduard Y. Chekmenev, b1977, PhD (supervisor Prof. Richard J. Wittebort) 2003, University of Louisville, KY, USA. Postdoctoral Fellow at NHMFL, Tallahassee, FL, USA (with Prof. Timothy Cross) and at Caltech (with Prof. Daniel P. Weitekamp) and HMRI (with Dr. Brian D. Ross). Co-authored $>70$ peer-reviewed original research publications in the areas of solidstate NMR of proteins and hyperpolarized NMR/MRI. Research interests include development of methods of hyperpolarization for Biomedical applications.

Keywords: Hyperpolarization, DNP, PHIP, Parahydrogen, Xenon, SABRE, Carbon-13, SEOP. 


\begin{abstract}
This chapter covers the fundamental principles and practice of NMR hyperpolarization techniques, which are proving useful for in vivo Magnetic Resonance Spectroscopy (MRS) studies of metabolism in animal models, and clinical trials with hyper-enhanced sensitivity. Fundamentally, hyperpolarization methods enhance nuclear spin polarization by orders-ofmagnitude, resulting in concomitant improvement in NMR detection sensitivity. The hyperpolarization methods described here-Dynamic Nuclear Polarization (DNP), Parahydrogen Induced Polarization (PHIP), Signal Amplification By Reversible Exchange (SABRE) and Spin-Exchange Optical Pumping (SEOP) — are capable of achieving nuclear spin polarization approaching the theoretical maximum of unity on nuclear spin sites of molecular or atomic agents suitable for in vivo administration. Importantly, hyperpolarization is inherently non-equilibrium in nature: the duration of the hyperpolarization is frequently short-lived, being limited by the in vivo spin-lattice relaxation times $\left(T_{1}\right)$ which are on the order of seconds to a minute. Nevertheless, sufficient amounts of nuclear spin polarization can survive the process of preparation, administration, and in vivo circulation to provide extraordinary enhancement of the hyperpolarized agent. The chemical shift dispersion of these agents at the molecular location of interest reports on functional, metabolic and other processes at the molecular level, enabling true molecular MRS imaging.
\end{abstract}




\section{INTRODUCTION}

The sensitivity and signal-to-noise ratio (SNR) of the Nuclear Magnetic Resonance (NMR) experiment is directly proportional to the nuclear spin polarization factor $P$, which represents the fraction of the alignment of the nuclear spin ensemble with an applied magnetic field $B_{0}$. In conventional NMR, $P \approx \gamma \hbar \mathrm{B}_{0} / 2 \mathrm{kT}$ under thermal equilibrium conditions governed by the Boltzmann distribution (with $\gamma=$ the gyro magnetic ratio; $\hbar=P l a n k$ ' $s$ constant $/ 2 \pi ; \mathrm{k}=$ Boltzmann's constant; and T=absolute temperature). In vivo conditions imply temperatures in excess of $300 \mathrm{~K}$. As a result, even if a high-field magnet-e.g. a 3 Tesla (T) clinical MRI scanner-is employed for magnetic resonance (MR) spectroscopy (MRS), the value of $P$ of proton $\left({ }^{1} \mathrm{H}\right)$ and carbon-13 $\left({ }^{13} \mathrm{C}\right)$ nuclear spins is only $1 \times 10^{-5}$ and $2.5 \times 10^{-6}$ respectively. Therefore, the vast majority of the nuclear spin ensemble does not in fact contribute to the NMR signal. For this reason, NMR in general and MRS in particular are frequently considered insensitive probes.

However, $P$ can be artificially increased significantly relative to the thermal equilibrium level, via a process called NMR hyperpolarization. Several hyperpolarization techniques realize enhancements of $P$ by several orders-of-magnitude over the Boltzmann level, and allow $P$ to approach the theoretical maximum of unity. The corresponding enhancement factor, $\varepsilon$, is field-dependent and defined as a ratio of $P$ in the hyperpolarized (HP) state to that in the thermal-equilibrium state (e.g. $\varepsilon=10^{4}$ to $10^{5}$ for ${ }^{13} \mathrm{C}$ at $\left.3 \mathrm{~T}\right){ }^{1}$

The fundamental concept of NMR hyperpolarization and its use in MRS is attractive because it offers extraordinary advantages over conventional MRS. ${ }^{2}$ First, the SNR can be significantly improved even using much lower concentrations of detected compounds than are present endogenously. Second, the data acquisition time can be dramatically decreased from tens of minutes down to sub-second scans. Third, an HP contrast agent (HCA) entering the biological system (i.e., excised organ, living organism, etc.) and reporting on metabolic 
functional events can provide insightful dynamic information on in vivo metabolism in real time compared to the static picture typically obtained with conventional single or multi-voxel MRS. Not surprisingly, biomedical applications represent the major driving force behind the fields of NMR hyperpolarization and HCA development, owing to their potential for revolutionizing medical diagnostics, drug therapy development, and probing physiological mechanisms.

However, HP NMR techniques are no panacea, as they have inherent disadvantages and shortcomings that must frequently be confronted during HCA production. First, HCAs are nearly always prepared exogenously in a hyperpolarization apparatus, frequently requiring sophisticated and expensive hardware. Second, HCAs must retain a HP state sufficiently long for quality assurance, transport, administration, in vivo delivery and NMR/MRS measurement. These requirements limit the number of molecular sites (i.e. moieties of ${ }^{13} \mathrm{C}$, ${ }^{15} \mathrm{~N},{ }^{129} \mathrm{Xe}$, etc.) suitable for HP MRS, to those whose spin lattice relaxation times $\left(T_{1}\right)$ are long enough to accomplish all this. Finally, the decay of the HP state to thermal equilibrium is irrecoverable. Therefore, signal acquisition must be fast and efficient to account for polarization losses via $T_{1}$ relaxation, RF pulsing (for detection), and the effects of in vivo metabolism and dilution. ${ }^{3}$

This chapter describes four hyperpolarization techniques: (1) Dynamic Nuclear Polarization (DNP), (2) Parahydrogen Induced Polarization (PHIP), (3) Signal Amplification By Reversible Exchange (SABRE) and (4) Spin-Exchange Optical Pumping (SEOP) of ${ }^{129} \mathrm{Xe}$. These techniques have already proven effective for preparing HCAs that can be used for tracking metabolic and functional processes in vivo (see emrstm $\left.1253^{3}\right)$, because they are capable of achieving $P$ approaching the theoretical maximum of unity on nuclear spin sites of molecular or atomic HCAs. Moreover, the HCAs for MRS fundamentally offer a wide dynamic range of chemical shift dispersions either through metabolism (e.g. ${ }^{13} \mathrm{C}$ metabolites) 
or by their sensitivity to the local environment (e.g. ${ }^{129} \mathrm{Xe}$ ). Many HCAs have been validated in animal models, ${ }^{2}$ while some HCAs (e.g. HP ${ }^{13} \mathrm{C}$-pyruvate and ${ }^{129} \mathrm{Xe}$ ) are being tested in clinical trials. $^{4}$

The primary focus in this chapter is on the fundamentals of these hyperpolarization techniques (i.e. how they are generated and their physical and chemical properties), their existing or emerging MRS relevance and a description of the hyperpolarization equipment (frequently referred to as hyperpolarizers) required to perform these techniques. Accordingly, the chapter describes and cites only selected works in these areas, and the reader may additionally benefit from some recent comprehensive reviews. ${ }^{2,5}$

\section{DYNAMIC NUCLEAR POLARIZATION (DNP)}

\subsection{Fundamentals}

Despite the demonstration by Overhauser in the early 1950s of the underlying basis of DNP — that $P$ could be enhanced by cross-relaxation with nearby electrons due to differences in their gyromagnetic ratios $\left(\gamma_{\mathrm{e}} / \gamma_{\mathrm{I}} \sim 660\right)$ - only recently has interest in its potential applications for biomedical MRI and MRS emerged. ${ }^{10}$ While various methods fall under the DNP umbrella, the "dissolution DNP" (d-DNP) method is the most commonly used method for in vivo applications. ${ }^{1,9}$ In biological d-DNP MRS experiments, the general preparation procedure involves: (i) mixing the MR-active species of interest with a paramagnetic source of free electrons; (ii) placing the mixture in a high magnetic field at low cryogenic temperatures; (iii) subsequent irradiation with a microwave source resonant on the electron spin resonance (ESR) frequency to mediate the transfer of electron polarization to nearby nuclei $^{11}$ followed by (iv) rapid sample thawing using a hot solvent to warm the frozen polarized sample and transfer to the MR scanner for in vivo injection as a liquid. These conditions generate electron spin polarizations that can approach unity as shown in Figure 1, 
with a significant fraction of the prepared $P$ largely surviving the rapid dissolution in step (iv).

$<$ Figure 1 near here $>$

$<$ Figure 2 near here $>$

\subsection{Dissolution DNP (d-DNP) Instrumentation}

Both commercial and home-built d-DNP devices have been extensively used for in vivo MRS. While each polarizer design may have unique characteristics, they are all comprised of the same basic components: a superconducting magnet that provides a $B_{0}$ of 3-7 T, a liquid helium cryostat for sample cooling to $1-4 \mathrm{~K}$, a microwave source to transfer polarization from the electrons to nuclei $(\sim 100 \mathrm{~mW})$, and a mechanism that rapidly dissolves the frozen sample pellet and delivers the solution for collection. ${ }^{9}$ Step (i) of the typical d-DNP experiment proceeds as follows: the species of interest is mixed with a radical source and a glassing agent in a typically flat sample container (up to a few hundred $\underline{\square}$ ) and lowered into the cryostat, as depicted in Figure 2. There in step (ii), the sample is frozen into an amorphous solid at low temperatures either by submersion into a liquid helium bath (where vacuumpumping reduces the vapor pressure, and hence bath temperature) or by exposure to a stream of continuously flowing liquid helium. The microwave source, whose frequency is set to the ESR line of the chosen radical species at the $B_{0}$ of the polarizer (typically within $\sim 80-140$ $\mathrm{GHz}$, with a $\sim 0.5 \mathrm{GHz}$ sweep range), irradiates the sample for $\sim 1 \mathrm{hr}$ in step (iii), using an antenna that takes advantage of the large flat surface area of the sample container. An onboard NMR system can check the HP signal while sweeping the microwave source to allow for optimization of the microwave frequency and excitation power (Figure 1c), and for monitoring the build-up of hyperpolarization over time (Figure 1d). 
Step (iv) of the procedure requires a superheated solvent with high heat capacity, which is typically satisfied by $\mathrm{pH}$-buffered saline. This is injected into the sample cup inside the cryostat. The lifetime of the HP signal is determined by the $T_{1}$ of the HCA, so to mitigate $P$ losses, the rapid dissolution is performed at high $B_{0}$ to minimize the sudden depolarizing effects of the paramagnetic centers as the temperature rises above $\sim 4 \mathrm{~K}$. Dissolution also reduces the relative concentration of radicals and lowers their contribution to the nuclear spin relaxation rate $^{9}$ as the sample volume expands by an order of magnitude (this process also dilutes the HP agent). The room-temperature (RT) $T_{1}$ values of most HCAs used for biomedical MRS are $\leq 1$ min but $B_{0}$ changes between the polarizer and MR scanner may further reduce $T_{1}$. Rapid shuttling of the sample from the polarizer to the scanner is key, and is typically performed pneumatically using helium gas through small diameter tubing. There, the polarized solution is quickly filtered to remove persistent radicals using anion-exchange or polyethylene filters ${ }^{9}$ and injected into the subject (Figure 2).

A commercially available d-DNP device for clinical use has recently been developed (SPINlab; GE Healthcare) that operates at $5 \mathrm{~T}$ and $1.1 \mathrm{~K}^{12}$ The system uses an on-board helium recycling system that dramatically lowers the consumption of liquid helium, which is expensive. While many other features of its design and operation are similar to that described above, additional quality-control steps are incorporated to ensure that the injectable solution meets clinical criteria for $\mathrm{pH}$, temperature, $P$ value, sterility, purity and residual radical specie concentration. In general, the larger sample volumes required for human use necessitate longer dissolution, transport, quality-control, and injection periods, such that significant time (e.g. $>65$ s) may elapse between the initial dissolution and final injection. Nevertheless typical ${ }^{13} \mathrm{C} P$ values are $\sim 15-20 \%$ after quality control, which is certainly sufficient to perform HP MRI/MRS in human patients with great advantage vs. conventional MRS with $P$ $\sim 10^{-3} \%{ }^{4}$ 


\subsection{Common HP agents produced with d-DNP}

\subsection{1 ${ }^{13}$ C-labelled small biomolecules}

The overall $P, T_{1}$, and biological relevance of the agent are the main criteria for choosing a HP species for MRS applications. Further considerations include the ability to form a glassy matrix in a sufficiently high concentration to mitigate dilution effects from the dissolution step; sufficient chemical shift range to differentiate the primary polarized species from any downstream products; the cost of isotopic enrichment; the ability to lessen the depolarization effects of nearby protons via deuteration; ${ }^{13}$ and toxicity. While a number of MR-active nuclei are eligible for DNP, the most commonly pursued are ${ }^{13} \mathrm{C}$-labelled carboxyl sites of metabolites. Of the latter, ${ }^{13} \mathrm{C}$-pyruvate has garnered the most attention because of its relatively high $P$ values $(>50 \%)$, long $T_{1}(\sim 60 \mathrm{~s})$, and ability to probe the tricarboxylic acid (TCA) cycle and glycolysis within a $T_{1}$-relevant timescale. ${ }^{14}$ Additional notable ${ }^{13} \mathrm{C}$-labelled small molecules pursued for DNP studies include urea, ${ }^{1}$ fumarate, glutamine, fructose, lactate, glucose, diethyl succinate, and acetate. ${ }^{2,13}$

\subsubsection{Extension to other nuclei}

Beyond ${ }^{13} \mathrm{C}$, the most commonly pursued d-DNP species for MRS studies are biologically relevant ${ }^{15} \mathrm{~N}$-labelled compounds, such as urea and choline. However, despite some benefits (the $T_{1}$ of ${ }^{15} \mathrm{~N}$-choline $\sim 4 \mathrm{~min}$ ), ${ }^{13}$ the lower gyromagnetic ratio makes ${ }^{15} \mathrm{~N}$ MRS more challenging for achieving useful signal intensities and gradient strengths for spatial localization with typical scanner hardware. Other species that can be hyperpolarized by DNP and may have potential for future in vivo applications include: ${ }^{1} \mathrm{H},{ }^{6} \mathrm{Li},{ }^{29} \mathrm{Si},{ }^{31} \mathrm{P},{ }^{89} \mathrm{Y},{ }^{107} \mathrm{Ag}$, and ${ }^{129} \mathrm{Xe}$.

\subsubsection{Secondary and co-polarization}

The enhanced signal from isotopically labeled HP small molecules may be translated into their chemical reaction products. This process, referred to as secondary polarization, allows 
the study of biologically relevant molecules that may not polarize well on their own, but have metabolic predecessors that do. This process can either be enzymatically regulated, such as with $\left[1,1-{ }^{13} \mathrm{C}_{2}\right]$ acetic anhydride breaking into different $\left[1-{ }^{13} \mathrm{C}\right] \mathrm{N}$-acetylated amino acids, ${ }^{13}$ or non-enzymatically regulated-for instance, the reaction of $1,2-{ }^{13} \mathrm{C}$-pyruvate with $\mathrm{H}_{2} \mathrm{O}_{2}$ to form ${ }^{13} \mathrm{CO}_{2}$, ${ }^{13} \mathrm{C}$-acetate, and ${ }^{13} \mathrm{C}$-bicarbonate. ${ }^{15}$ Co-polarization of a single "batch" of different small molecules using d-DNP during agent production allows the simultaneous study of different biophysical parameters and processes such as $\mathrm{pH}$, metabolism, necrosis, and perfusion. ${ }^{13}$ While the commingled molecules' solid-state polarization $P$ and $T_{1}$ values are often similar to their individual values, drawbacks include lower concentrations of, and potential interactions among, the small molecules involved.

\subsection{Radicals and Glassing Agents}

\subsubsection{Choice of radical species}

The build-up time and hyperpolarization level for all species are highly dependent on the choice of radical used to mediate the DNP. Typical radical concentrations range from 15-60 $\mathrm{mM}$, and may persist post-thawing, requiring filtering before administration. The most widely used sources of free electrons for ${ }^{13} \mathrm{C}$ DNP have been the trityl and 1,3bisdiphenylene-2-phenylallyl (BDPA) radicals. Because their ESR lines are narrower than the ${ }^{1} \mathrm{H}$ resonance frequency dispersion, the probability of cross-polarization with ${ }^{1} \mathrm{H}$ is lowered, which increases the chances of polarizing nearby ${ }^{13} \mathrm{C}$ nuclei.

Further improvements in the $P$ level and reductions in buildup time can be gained by the addition of a $\mathrm{Gd}^{3+}$ complex to the trityl radical solutions. The highly efficient electron- ${ }^{1} \mathrm{H}$ cross-polarization of nitroxyl radicals (e.g., TEMPO; 2,2,6,6-tetramethylpiperidine-1-oxyl) can also be used in conjunction with a ${ }^{1} \mathrm{H}_{-}{ }^{13} \mathrm{C}$ cross-polarization scheme to decrease ${ }^{13} \mathrm{C}$ polarization time, albeit with lower overall ${ }^{13} \mathrm{C}$ polarization compared to trityl or BDPA radicals. Liquid-state $P$ can be further increased using a deuterated solvent for the nitroxyl 
radicals. ${ }^{9}$ Additional gains have been reported using bi-radicals comprised of two TEMPO radicals tethered by a polyethylene chain. ${ }^{16}$

\subsubsection{Choice of glassing agent}

The DNP process is most efficient when the HP molecule is homogenously mixed with the radical species and formed into a glass, as already noted. This amorphous state allows the paramagnetic centers to achieve close contact with nearby nuclei, which is not the case in a crystalline lattice. Typical glassing agents include glycerol and dimethyl sulfoxide (DMSO), which act to prevent crystallization. Some HP molecules such as neat ${ }^{13} \mathrm{C}$-pyruvate, are capable of self-glassing ${ }^{17}$, which allows for a higher concentration of ${ }^{13} \mathrm{C}$ nuclei that speeds

${ }^{13} \mathrm{C}$ spin diffusion resulting in a more homogenously-polarized sample. ${ }^{18}$

\subsection{DNP Advantages, Limitations, and Future Prospects}

As a hyperpolarization method, DNP's versatility lies in its ability to hyperpolarize a variety of biologically relevant molecules not easily polarized by other methods. Target molecules can achieve high $P$ with sufficiently long $T_{1}$ values for probing metabolic pathways, and clinical trials are already underway for $\mathrm{HP}{ }^{13} \mathrm{C}$-pyruvate. The main limitations thus far are the high cost of purchase, maintenance, and infrastructure required for the polarizers. Improvements in cryogen recovery and solid-state cooling can lower operational costs, and further optimization of the physical and chemical environment of the HP species should improve $P$ and $T_{1}$ values, as would the ability to store the enhanced polarization in a singlet state until it is ready for MRS applications. Finally, the demonstration of a clinically important application of HP MRI- such as using ${ }^{13} \mathrm{C}$-pyruvate to study prostate cancer or heart dysfunction — will greatly hasten the development of all d-DNP aspects. ${ }^{9}$

\section{PARA-HYDROGEN (para-H $)$ PRODUCTION}

In addition to its more recent applications in HP MRS, the properties of para-hydrogen (para $\left.-\mathrm{H}_{2}\right)$ have proved important for hydrogen's use as a rocket fuel by minimizing boil-off 
due to state conversion, and even earlier, $p a r a-\mathrm{H}_{2}$ served as a prototypical system during the development of quantum theory. The di-hydrogen molecule possesses two nuclear spin isomers, denoted as "ortho-" and "para-" states, comprised of a nuclear triplet state and a singlet state, respectively. Importantly, while representing the simplest nuclear singlet state, para $-\mathrm{H}_{2}$, per se, has net spin $I=0$ and is therefore NMR invisible. As will be seen in the following sections, its use in NMR/MRS is as an extremely powerful vector for transferring HP to HCAs of interest. For further discussion of the physics of parahydrogen, we direct the interested reader to the books by Farkas on hydrogen ${ }^{19}$ and Linus Pauling on quantum mechanics ${ }^{20}$, and the review by Green and co-workers. ${ }^{5}$

Inter-conversion of ortho-hydrogen (ortho- $\left.\mathrm{H}_{2}\right)$ to para $-\mathrm{H}_{2}$ (with a heat of conversion of $670 \mathrm{~J} / \mathrm{g}$ ) is forbidden according to the selection rules of quantum mechanics. It thus occurs very slowly. Spontaneous emission of radiation was calculated by Wigner to result in a conversion rate of $\sim 10^{-10} \mathrm{sec}^{-1}$, or approximately one transition per 1 gram every three hundred years. ${ }^{19}$ In 1930, Hall and Oppenheimer calculated that molecular collisions at atmospheric pressure result in a rate of one per $\sim 10^{8} \mathrm{sec} / 1 \mathrm{~g}$ for the ortho-para transition half lifetime, or about 3 years. ${ }^{19}$ While such a slow inter-conversion rate is nominally unfavorable to para $-\mathrm{H}_{2}$ production, Bonhoeffer and Harteck discovered in 1929 that paramagnetic catalysts, including for example, activated charcoal, nickel, and hydrated iron-III oxide, greatly accelerate the establishment of thermodynamic equilibrium for the ratio of ortho$\mathrm{H}_{2} /$ para $-\mathrm{H}_{2}$.

$<$ Figure 3 near here $>$

The instrumentation used to generate high-purity para-state dihydrogen is commonly called a "parahydrogen generator," shown in Figure 3. The essential purpose of the generator is to pass bulk $\mathrm{H}_{2}$ at RT (i.e. $\sim 293 \mathrm{~K}$ ) over a paramagnetic catalyst at a cryogenic temperature of $\sim 20 \mathrm{~K}$ to rapidly equilibrate the $75 \%$ ortho $-\mathrm{H}_{2}$ fraction to the lower-temperature 
Boltzmann thermal equilibrium, thereby creating para $-\mathrm{H}_{2}$ with nearly $100 \%$ para-fraction. Typically, the gas flows through a catalyst chamber attached to a two-stage, Joule-Thomson effect, helium cryocooler cold-head. The flow is regulated with either: (i) flow restrictors such as mass flow controllers, snubbers, or needle valves; ${ }^{21,22}$ or (ii) by releasing small batches of compressed $\mathrm{H}_{2}$ between automatically-sequenced solenoid valves. ${ }^{23}$ After separation of the para- $\mathrm{H}_{2}$ from the catalyst and subsequent storage at $\mathrm{RT}$, quantummechanical symmetry selection rules forbid re-establishment of the RT equilibrium. Provided the para $-\mathrm{H}_{2}$ storage vessel contains no paramagnetic impurities (as is the case for those glasslined or of aluminum, although the absence of $\mathrm{O}_{2}$ is also important), the relaxation rate of the para-hydrogen back to the RT equilibrium can be on the order of months. ${ }^{21-23}$ Thus para- $\mathrm{H}_{2}$ experiments are possible long after production.

\section{PARA-HYDROGEN INDUCED POLARIZATION (PHIP)}

\subsection{Fundamentals}

While the para- $\mathrm{H}_{2}$ molecule is itself NMR invisible, Bowers and Weitekamp ${ }^{24}$ have demonstrated that the para $-\mathrm{H}_{2}$ singlet can be unlocked via the chemical reaction of hydrogenation provided that the para- $\mathrm{H}_{2}$ is added in a pairwise manner (best exemplified in the molecular mechanism of $\mathrm{H}_{2}$ addition), and that the nascent protons in the resulting hydrogenation product are no longer magnetically equivalent. This phenomenon of symmetry breaking the para- $\mathrm{H}_{2}$ nuclear singlet state via hydrogenative processes is termed "parahydrogen induced polarization" (PHIP). ${ }^{25}$

$<$ Figure 4 near here $>$

While the para- $\mathrm{H}_{2}$ singlet can be preserved for weeks and months, once the symmetry is broken and the para- $\mathrm{H}_{2}$ pair is incorporated into the molecular framework of a product molecule (Figure 4), the relaxation decay processes (via $T_{1}$ and the spin-spin relaxation time, 
$T_{2}$ ) are significantly more efficient (on a time-scale of seconds) —leading to rapid depolarization. For the majority of metabolically relevant injectable contrast agents that can be envisioned, fast $T_{1}$ relaxation limits any meaningful preparation of HCA for biomedical use.

This initial fundamental PHIP challenge was overcome in the early 2000 s, when an adjacent ${ }^{13} \mathrm{C}$ carboxyl (and potentially ${ }^{15} \mathrm{~N}$ or others) with significantly longer $T_{1}$ was introduced by Golman ${ }^{26}$ and Goldman. ${ }^{27}$ In addition, deuteration of the PHIP molecular precursor can extend the $T_{1}$ lifetime of the nascent protons, which has proven especially useful in PHIP of gases. ${ }^{28}$ In the PHIP process, polarization from nascent para- $\mathrm{H}_{2}$ protons is efficiently transferred to the ${ }^{13} \mathrm{C}$ nucleus via the network of $J$-couplings using either magnetic field-cycling or radiofrequency (RF) pulse-based methods. Regardless of the approach used, the chemical reaction between para- $\mathrm{H}_{2}$ and the unsaturated substrate molecule must be performed on a relatively fast time scale (seconds), i.e. significantly shorter than the effective relaxation times of the nascent para- $\mathrm{H}_{2}$ protons, in order to minimize polarization losses during the reaction. Post-reaction, the polarization is typically transferred to the ${ }^{13} \mathrm{C}$ nucleus within a second. Moreover, even in the case of RF-based polarization transfer, which has proven to be more efficient, as gauged by the $\% P$ delivered to ${ }^{13} \mathrm{C}$, to date, the PHIP method is relatively low cost because the PHIP process can be performed at very low magnetic fields (a few mT) using relatively simple NMR hardware.

\subsection{Molecular Precursors and Contrast Agents}

The design of PHIP precursor molecules for biomedical applications and MRS is a challenging process that must meet critical requirements. In particular, the molecule must possess an unsaturated (typically $\mathrm{C}=\mathrm{C}$ ) bond adjacent to ${ }^{13} \mathrm{C}$ to provide a $J$-coupling contact between the nascent para- $\mathrm{H}_{2}$ protons and the ${ }^{13} \mathrm{C}$ to enable efficient polarization transfer. Furthermore, the efficiency of this polarization transfer in RF pulse-based polarization 
transfer methods can greatly benefit from deuteration of the precursor molecule ${ }^{29}$ as a means to keep the nuclear spin system effectively constrained to just the three involved spins: the two nascent parahydrogens and the ${ }^{13} \mathrm{C}$ nucleus. ${ }^{27}$ Lastly, because the hydrogenation reaction may be incomplete, both the precursor and product molecules should have relatively low in vivo toxicity. Meeting all these requirements represents the main disadvantage of PHIP, and only a limited number of potentially amenable precursor molecules and HCAs have qualified for in vivo use, so far.

Those that have been successfully hyperpolarized and used for in vivo MRS include HP ${ }^{13} \mathrm{C}$-succinate, which highlights metabolites of the TCA cycle ${ }^{30} ;{ }^{13} \mathrm{C}$-tetrafluoropropyl propionate, for sensing lipids in atherosclerotic plaques, ${ }^{31}$ and ${ }^{13} \mathrm{C}$-phospholactate ${ }^{29}$ for preparation of $\mathrm{HP}{ }^{13} \mathrm{C}$-lactate, which has been shown to be useful for in vivo metabolic imaging of elevated glycolysis in tumors.

\subsection{Homo- and Heterogeneous PHIP Catalysis}

\subsubsection{Homogeneous Catalysis in Organic Solvents}

PHIP $^{24,25}$ has attracted the attention of those working in catalysis due to its promise to increase the NMR sensitivity of key low-concentration species. The PHIP process of pairwise para $-\mathrm{H}_{2}$ addition requires an efficient catalyst to complete the process within seconds (i.e. on the time-scale of relaxation processes). Initially, PHIP catalytic systems were based on Wilkinson's catalyst $\mathrm{RhCl}\left(\mathrm{PPh}_{3}\right)_{3},{ }^{24}$ Vaska's complex $\operatorname{Ir}(\mathrm{CO}) \mathrm{Cl}\left(\mathrm{PPh}_{3}\right)_{2}$, and Crabtree's catalyst $\left[(\mathrm{COD}) \operatorname{Ir}\left(\mathrm{PCy}_{3}\right)(\mathrm{Py})\right]^{+} \mathrm{PF}_{6}^{-},\left(\mathrm{COD}=1,4\right.$-cyclooctadiene, $\mathrm{PCy}_{3}=$ tricyclohexylphosphine, $\mathrm{Py}=$ pyridine). These transition metal-based systems catalyze the molecular hydrogenation reaction, where para $-\mathrm{H}_{2}$ is added to an unsaturated precursor via a molecular mechanism. Undergoing a molecular rather than a radical mechanism is essential for preserving the para- $\mathrm{H}_{2}$ spin order, which allows the observation of a PHIP effect. ${ }^{5,24} \mathrm{~A}$ number of other transition metals and chelating ligands have been evaluated. ${ }^{5}$ In particular, 
implementation of bidentate ligands such as $\mathrm{Ph}_{2} \mathrm{P}\left(\mathrm{CH}_{2}\right)_{4} \mathrm{PPh}_{2}$ using $\mathrm{Rh}(\mathrm{I})$ as a catalyst has proven to be particularly effective for the ultrafast molecular addition of $\mathrm{para}-\mathrm{H}_{2}$ to double and triple bonds, resulting in polarization enhancements of $\sim 10^{4}$ upon transfer to adjacent ${ }^{13} \mathrm{C}$ nuclei. ${ }^{26}$ Despite the apparent synthetic success of this methodology, the approach has been limited to organic solvents, thus limiting biological applications where aqueous catalysts and substrates are desired.

\subsubsection{Homogeneous Aqueous Catalysis}

Chemical modification of phosphine-based ligands has permitted preparation of efficient water-soluble $\mathrm{Rh}(\mathrm{I})$-based catalytic systems. By far, the most common variation utilizes 4bis[(phenyl-3-propanesulfonate) phosphine $]$ butane di-sodium or its close variant, ${ }^{22}$ where $\mathrm{Rh}(\mathrm{I})$ is chelated by norbornadiene and two phosphines connected by a four- or three- carbon bridge. ${ }^{5}$ While aqueous homogeneous catalysts have opened the door to the first generation of mammalian in vivo imaging aided by $\mathrm{PHIP}^{30,31}$, the presence of $\mathrm{Rh}(\mathrm{I})$ catalysts in the HCA solution is presently an obstacle to clinical applications. Replacement of homogeneous PHIP by heterogeneous PHIP (“HET-PHIP”) processes will likely be the key to PHIP clinical applications. HET-PHIP catalysis was extensively reviewed a few years ago, ${ }^{32}$ and is briefly discussed here in the context of recent advances and applications for aqueous media.

\subsubsection{Heterogeneous (Solid/Liquid) Catalysis}

A number of immobilized Rh complexes, based on either Wilkinson's catalyst or closely related systems based on $\mathrm{Rh}(\mathrm{I})$ derived from $\left[\mathrm{Rh}(\mathrm{COD})_{2}\right]^{+}\left[\mathrm{BF}_{4}\right]^{-}$, have been evaluated for PHIP, typically using diphenylphosphinoethyl-modified silica as a stationary phase. While some PHIP signal was often observed, the enhancement values were generally low due to slow hydrogenation rates, loss of para $-\mathrm{H}_{2}$ polarization on the stationary phase surfaces, and chemical degradation of the catalyst. Alternatively, the application of transition metal nanoparticles is well-established for gas-solid PHIP (see below), and has been demonstrated 
for capped platinum nanoparticles ${ }^{33}$ in liquid-solid PHIP, where HP liquids are generated over solid-phase catalysts. Finally, $\mathrm{Rh} / \mathrm{TiO}_{2}$ nanoparticles have also been used for PHIP processes, and PHIP hyperpolarization of acrylamide and allyl methyl in $\mathrm{D}_{2} \mathrm{O}$ solutions was demonstrated using these $\mathrm{Rh} / \mathrm{TiO}_{2}$ nanoparticles. This approach is promising for in vivo applications in spite of the low $P$ levels demonstrated to date.

\subsubsection{Heterogeneous (Solid/Gas) Catalysis}

A silica-embedded Wilkinson's catalyst was also the first catalytic system enabling PHIP of gases at polarization levels sufficient for in vitro imaging. ${ }^{34}$ Further progress in this area has been driven by the development of more robust nanoparticle catalysts such as: Pt, Pd or Rh on $\mathrm{TiO}_{2}, \mathrm{SiO}_{2}, \mathrm{Al}_{2} \mathrm{O}_{3}$, or $\mathrm{ZrO}_{2}$ solid substrates. In general, better polarization transfer results were obtained with smaller nanoparticles: Rh and Pt provide superior results to Pd, while $\mathrm{Al}_{2} \mathrm{O}_{3}$ and especially $\mathrm{TiO}_{2}$ were superior performing substrates. ${ }^{32}$

\subsection{Hyperpolarization Instrumentation}

The controlled reaction of para- $\mathrm{H}_{2}$ with an unsaturated chemical precursor to produce a HCA takes place within an instrument referred to as a 'PHIP hyperpolarizer'. The features and underlying polarization transfer technique differentiate the hyperpolarizers constructed to date. $^{26,35,36}$ In general, inert gases such as $\mathrm{N}_{2}$ shuttle the precursor through a manifold during injection into a heated reactor wherein it is rapidly and thoroughly mixed with a para- $\mathrm{H}_{2}$ atmosphere, as shown in Figure 5. Prior to injection, the precursor is typically pre-heated to further speed the chemical reaction of pair-wise addition. Two approaches to precursor/para$\mathrm{H}_{2}$ mixing have involved: (i) jet entrainment mixing; ${ }^{22}$ and more commonly (ii) spray mixing (Figure 5). ${ }^{26,35,36}$ Other differences in hyperpolarizer designs concern the method used to transfer the spin order of para- $\mathrm{H}_{2}$ after chemical synthesis to the heteronucleus which include: (i) field-cycling; ${ }^{26}$ or (ii) more efficiently, ${ }^{26,35,36}$ the application of RF pulses. ${ }^{27}$ In the latter method, RF pulses may be synchronized with the operation of the manifold by a 
LabView interface to a National Instruments data acquisition card, ${ }^{26,35,36}$ or by a low-field NMR spectrometer. ${ }^{36}$ In order to facilitate calibration of the RF pulse sequence parameters, and in situ NMR detection capability for the hyperpolarizer is desirable. ${ }^{36}$ The latter can result in a stand-alone PHIP hyperpolarizer with built-in quality assurance for the $P$ produced.

$<$ Figure 5 near here $>$

\section{SIGNAL AMPLIFICATION BY REVERSIBLE EXCHANGE}

\section{(SABRE)}

Another route to achieve hyperpolarization in solution is SABRE (Signal Amplification by Reversal Exchange), introduced by Duckett and co-workers in $2009 .{ }^{37}$ Similar to traditional PHIP, SABRE also takes advantage of the transfer of the spin order of para- $\mathrm{H}_{2}$ to a target substrate brought together by an organo-metallic catalyst. The key difference is that with SABRE, the transfer of spin order is not dependent upon the hydrogenation of an unsaturated bond of the substrate. This difference brings about two advantages: (i) the expansion of the range of applications of $\mathrm{PHIP}^{24,25}$ with respect to the types of molecules that can serve as substrates; and (ii) that destruction or alteration of the original structure of those substrates is not required — one does not need to develop a precursor structure that will yield a desired agent upon hydrogenation. SABRE is the newest of the hyperpolarization techniques described in this Article, and consequently, its development for MRS/MRI is less advanced. It has some intrinsic advantages including simplicity, low cost, scalability, and the potential for continuous agent production, which are promising for biomedical applications. Indeed, it has already achieved substantial polarization enhancements in solution, with polarizations reaching $\sim 10 \% .^{38-40}$ 
In order for SABRE to work, the catalyst must transiently bind both the target substrate and the atoms of para- $\mathrm{H}_{2}$ with residence times that are long enough to allow the transfer of spin order or polarization to occur, but short enough to allow rapid turnover and subsequent accumulation of HP substrate in the solution. The process is illustrated in Figure 6. Although multiple organometallic complexes have been tested for SABRE, all to date use an iridium atom at the center-including the most successful SABRE catalyst, $\operatorname{IrCl}(\mathrm{COD})(\mathrm{IMes})$, where IMes $=1,3$-bis(2,4,6-trimethyl-phenyl)imidazol-2-ylidene, and COD = cyclooctadiene. While the latter is not commercially available, its preparation is straightforward for those experienced with synthesis in inert-atmosphere environments. ${ }^{41}$

$<$ Figure 6 near here $>$

As with many catalysts used in traditional PHIP, the basic iridium-based SABRE catalyst must first be activated before use. This typically involves dissolving the catalyst in an organic solvent along with excess substrate such as pyridine or a structural derivative: attempting to activate the catalyst without excess substrate can lead to irreversible de-activation of the catalyst. ${ }^{42}$ The solution is charged with para- $\mathrm{H}_{2}$, either by loading a few atm of para- $\mathrm{H}_{2}$ over the sample within a pressure-resistant vessel, or by continuously bubbling the para- $\mathrm{H}_{2}$ within a specially designed apparatus/tube, such as that depicted in Figure 7. Step-wise hydrogenation of the molecular COD-ring and binding of the $\mathrm{H}_{2}$ to the Ir leads to loss of the eight-carbon ring, and the formation of a six-coordinate site complex, with ligands comprising the IMes group, two hydrides, and three substrates, two of which are exchangeable. The progress of activation may be followed by observing changes in the characteristics of hydride resonances of transient species and the final activated structure. ${ }^{42}$ $<$ Figure 7 near here $>$

SABRE experiments can be divided into two types: ex situ and in situ, depending on the location of the sample vessel during polarization transfer with respect to the MR magnet. 
The vast majority of SABRE experiments are performed ex situ: spin order is transferred via scalar couplings between the involved nuclear spins during the lifetime of the catalyst complex. The spin flips must be nearly energy-conserving: thus, the magnetic field must be sufficiently low to make the frequency difference between the hydride spins and the substrate spins similar to the scalar coupling between them. This corresponds to $\sim 6 \mathrm{mT}$ for ${ }^{1} \mathrm{H}$ and $\sim 1 \mu \mathrm{T}$ for ${ }^{15} \mathrm{~N} .{ }^{40}$. Thus the sample must be outside the MR magnet or within a fringe field for polarization transfer, and the sample rapidly moved to the magnet for detection. However, SABRE enhancements can also be observed in situ at high field. ${ }^{41}$ In this case, the mechanism appears to be mediated by dipolar cross-relaxation between the HP hydride spins and the substrate spins. ${ }^{41,42}$ While much less efficient, in situ SABRE does not require sample shuttling. In addition, the application of RF pulse sequences, for example, to allow mixing of spin states by driving the spins into level anti-crossing regimes, ${ }^{43}$ may dramatically improve the efficiency of SABRE in situ.

To date, only a limited number of HCAs have been hyperpolarized via SABRE. Besides pyridine (with ${ }^{1} \mathrm{H}$ and ${ }^{15} \mathrm{~N}$ polarizations approaching $\sim 10 \%{ }^{38,40}$ ), SABRE enhancements of amino acids and small peptides have been reported at low field. ${ }^{44}$ Most attention has been directed at biologically relevant pyridine derivatives. For example, ${ }^{1} \mathrm{H}$ enhancements of $\sim 230$ and $\sim 1400$ vs. conventional MRS were reported for the tuberculosis drugs isoniazid and pyrazinamide in deuterated methanol at $700 \mathrm{MHz}$, respectively corresponding to ${ }^{1} \mathrm{H} P$ values $\left(P_{\mathrm{H}}\right)$ of nearly $\sim 1.3$ and $\sim 8 \% .{ }^{39}$ Nicotinamide was used to demonstrate SABRE in pure water, albeit with a more modest enhancement of $\sim 33$ fold at $9.4 \mathrm{~T}^{42}$ by first activating the catalyst in ethanol, drying it, and reconstituting it in an aqueous solution.

Finally, in addition to the limitations imposed by the catalysts and substrates that are presently available and the need to perform SABRE in biologically compatible media, the catalysts themselves present an obstacle to the application of SABRE in the clinic. The 
catalysts are expensive and potentially toxic, making their recovery desirable after agent hyperpolarization. In this regard, following efforts of heterogeneous PHIP (“HET-PHIP”) by Koptyug and co-workers, ${ }^{32}$ "HET-SABRE" enhancement of pyridine using SABRE catalysts that were covalently immobilized onto solid substrates of microscale polymer beads has been achieved. ${ }^{45}$ Although the enhancements were small ( $\sim 5$-fold $)$, the feasibility of creating separable catalysts for the creation of pure SABRE HP agents was demonstrated.

\section{SPIN-EXCHANGE OPTICAL PUMPING (SEOP)}

Hyperpolarization of noble gases via spin-exchange (SE) optical pumping (OP) was first demonstrated by Bouchiat et al. in 1960, following earlier pioneering work involving OP of alkali metal vapors by Kastler and others. ${ }^{46}$ Over time the technique has been refined with an aim to increase efficiencies of the OP and SE steps, as well as to reduce spin-relaxation or "spin-destruction" rates, to enable the production of HP gases with sufficiently high $P$ in large enough quantities for NMR/MRI applications. Major technical advances in these areas came with the implementation of high-power laser diode arrays (LDAs) ${ }^{47}$ and the introduction of buffer gases and cell coatings. ${ }^{46}$ These improvements have contributed to increases in noble gas polarization, including values as high as $\sim 90 \%$ for ${ }^{129} \mathrm{Xe}$ (e.g., Ref. ${ }^{48}$ ), upon which our discussion will focus.

Although the complexity of SEOP setups may vary, one minimally needs: (i) an OP "cell" that contains the noble gas of interest $\left({ }^{129} \mathrm{Xe}\right)$ and a small amount of alkali metal $(\mathrm{Rb}$ in the following discussion); (ii) a source of circularly polarized light resonant with the $\mathrm{Rb}$ electronic transition; (iii) a heater for the cell (to vaporize the Rb); and (iv) a magnetic field source. Circularly polarized laser light is used to optically pump $\mathrm{Rb}$ electrons into a selected spin state, as shown in Figure 8. A usually weak ( 30 Gauss) magnetic field provides hyperfine Zeeman splitting of the Rb electronic energy states. Angular momentum is then transferred from the $\mathrm{Rb}$ electrons to the ${ }^{129} \mathrm{Xe}$ nuclear spins (Figure $8 \mathrm{~b}$ ) via gas-phase 
collisions. ${ }^{46}$ Provided the $\mathrm{OP} / \mathrm{SE}$ rates respectively exceed the electron and nuclear spinrelaxation rates, ${ }^{129}$ Xe polarization can be increased far above thermal equilibrium according to ${ }^{49}$ :

$$
P_{X e}\left(t_{p}\right)=\frac{\gamma_{s e}}{\gamma_{s e}+\gamma_{w}}\left\langle P_{R b}\right\rangle\left(1-e^{-\left(\gamma_{s e}-\gamma_{w}\right) t_{p}}\right)
$$

where $P_{\mathrm{Xe}}$ and $P_{\mathrm{Rb}}$ represent the polarizations of xenon nuclei and rubidium electrons respectively, $\gamma_{\text {se }}$ is the spin-exchange rate, $\gamma_{\mathrm{w}}$ is the rate of ${ }^{129} \mathrm{Xe}$ spin destruction, and $t_{\mathrm{p}}$ is the polarization time. Also present is $\mathrm{N}_{2}$ as a buffer gas, which at a pressure of $\sim 100-200$ Torr is sufficient to collisionally quench alkali metal fluorescence, thus avoiding re-emission of photons with random polarization that would otherwise decrease $P_{\mathrm{Rb}} \cdot{ }^{46,50}$ One challenge for hyperpolarizing ${ }^{129} \mathrm{Xe}$ is that most $\mathrm{Rb} / \mathrm{Xe}$ collisions tend to depolarize $\mathrm{Rb}$ atoms without conserving spin angular momentum, making the $\mathrm{Rb}$ spin-destruction rate effectively proportional to the xenon nuclear density $[\mathrm{Xe}]$ —an issue that can be mitigated through the use of higher resonant laser fluxes (albeit with greater demands on thermal regulation ${ }^{50}$ ) and/or lower Xe partial pressures. Conducting SEOP for ${ }^{129} \mathrm{Xe}$ at lower total cell pressures also enables more efficient spin exchange through three-body van der Waals collisions, in addition to the binary spin exchange that is dominant at higher gas pressures. ${ }^{51}$ On the other hand, higher cell pressures can broaden the $\mathrm{Rb}$ spectral lines resulting in more efficient absorption of the light. ${ }^{49}$ Finally, silicon coatings are often used to reduce Xe nuclear spin destruction, and all the components of the SEOP setup must be chosen to minimize exposure of the HP gas to paramagnetic centers, strong field gradients, and zero-crossings of the magnetic field.

$<$ Figure 8 near here $>$

Many different polarizers have been developed to maximize the achievable polarization, $P_{\mathrm{Xe}}$, and/or the rate of production of HP Xe. Their designs are typically grouped into two categories. First, in the continuous-flow (CF) method illustrated in Figure $9^{49,51}$, a xenon gas 
mixture is polarized as it passes continuously through the OP cell and is subsequently collected using a cryogenic "cold finger", ${ }^{49}$ where it is stored at high field ( $\left.>500 \mathrm{G}\right)$ before it is carefully warmed ${ }^{52}$ to allow transfer to a secondary vessel with minimal losses of $P_{\text {Xe. }}$. For example, Ruset et al. (Figure 9a) employed a number of features - including a Rb presaturator upstream of a $\sim 1.8 \mathrm{~m}$ optical cell, a high laser power $(\sim 90 \mathrm{~W})$, a carefully designed optical path, and a relatively low Xe density and total pressure (allowing it to exploit the more efficient 3-body van der Waals spin-exchange interaction) — to attain a peak ${ }^{129} \mathrm{Xe}$

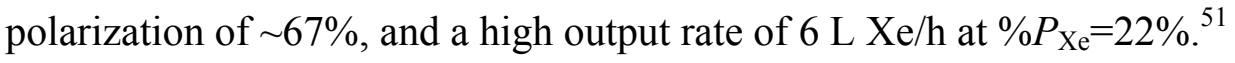

$<$ Figure 9 near here $>$

Second, the stopped-flow (SF) method (Figure $9 b^{48,53}$ ) takes place in a closed system where gas delivery, SEOP, and collection are performed sequentially. The cell is loaded with the desired gas mixture, and the gas is illuminated by the laser for $\sim 10-30 \mathrm{~min}$. until $P_{\mathrm{Xe}}$ nears a steady state. Prior to collection, the SEOP cell is cooled to condense the alkali metal vapor. The HP Xe can then be cryo-collected or simply expanded into another container (e.g. a Tedlar ${ }^{\circledR}$ bag).

The second approach is simpler and easier to automate, and the reduction in magnetization density that results from HP gas dilution with buffer gas can be mitigated by using high $[\mathrm{Xe}]$ mixes. ${ }^{48}$ Nikolaou et al. recently presented an 'open-source' SF polarizer design (Figure 9b-d ${ }^{54}$ ) utilizing a $200 \mathrm{~W}$ narrowed $(\sim 0.27 \mathrm{~nm}$ FWHM) LDA capable of attaining polarizations of $\sim 90 \%, \sim 57 \%, \sim 50 \%, \sim 30 \%$, for Xe partial pressures of $\sim 300, \sim 500$, $\sim 760$, and $\sim 1570$ Torr backfilled with $\mathrm{N}_{2}$ to 2000 Torr, with a throughput of $\sim 1 \mathrm{~L} / \mathrm{h} .{ }^{48}$

Standard diagnostic techniques used in SEOP experiments include in situ low-field NMR spectroscopy to determine and control for $P_{\mathrm{Xe}}$, and near-infrared optical absorption spectroscopy to monitor pump laser wavelength, absorption profile, and inferred electronic $P_{\mathrm{Rb}}$. While not strictly necessary for HP Xe preparation, additional diagnostic techniques 
such as: Faraday rotation; ${ }^{55} \mathrm{ESR}^{46}$ to measure alkali metal density and spin polarization; and Raman spectroscopy to map elevated gas temperatures within the OP cell, have also been implemented to better understand the complex processes underlying mass, energy, and polarization transport within the SEOP apparatus.

MRS applications of HP Xe typically exploit the extraordinary sensitivity of xenon's chemical shift to its local molecular environment (Figure $8 \mathrm{c}) .^{7}$ Of the two NMR-active xenon isotopes $-{ }^{129} \mathrm{Xe}(I=1 / 2)$ and ${ }^{131} \mathrm{Xe}(I=3 / 2)$ - only ${ }^{129} \mathrm{Xe}$ has sufficiently long relaxation times in condensed phases to allow MRS. Xenon's highly polarizable electron cloud also allows it to participate in relatively substantial van der Waals interactions with proteins, lipid membranes, and living tissues. Xenon is not naturally found in the body in appreciable concentrations, which is both an advantage and a limitation: $\mathrm{HP}{ }^{129} \mathrm{Xe}$ spectra will be background-free, but achieving efficient HP Xe delivery to the body is key. Delivery options are: respiration and dissolution into the blood from the lungs; direct or intravenous injection of Xe-saturated solutions; ${ }^{7}$ or ex vivo gas dissolution into the blood or other solutions using off-the-shelf gas-exchange modules. ${ }^{56}$

When Xe is dissolved in bodily tissues, a number of resonances corresponding to different cellular and tissue compartments may be observed generally within a range $\sim 185$ 215 ppm downfield from the gas reference at $\sim 0 \mathrm{ppm}^{7}$ For example, the shift of ${ }^{129} \mathrm{Xe}$ is so sensitive that its resonance in red blood cells responds to changes in blood oxygenation. ${ }^{57}$ Functional studies of the brain are thus possible. The large ${ }^{129} \mathrm{Xe}$ shift between xenon in the lung space and that dissolved in tissues may be exploited for a variety of pulmonary functional MRS/MRI studies, including studies of gas exchange efficiency in healthy versus pathological tissues. Nevertheless, without further intervention xenon's concentration in the body is generally too low — and its interactions too weak and non-specific - for ${ }^{129} \mathrm{Xe}$ MRS to provide much more in the way of specific biomolecular information. 
To overcome this low sensitivity and improve its specificity, HP ${ }^{129} \mathrm{Xe}$ can be augmented with a host molecule, which can be functionalized to act as a biosensor as exemplified in Figure $10 .^{58}$ The approach exploits the strong interaction between xenon and cryptophanes, which are molecules that can bind Xe atoms within their internal hydrophobic cavities. Indeed, cryptophanes are an excellent choice for the superstructure of xenon biosensors, not only because of their relatively high xenon affinity $\left(\mathrm{K}_{\mathrm{a}} \sim 10^{3}-10^{4} \mathrm{M}^{-1}\right)$, but also because the exchange and relaxation timescales are compatible with MRS. Moreover, they can be chemically functionalized to facilitate both aqueous solubility and covalent linkage to a chosen functional group to target specific bioanalytes. In principle, almost any ligand or antibody can be used as a functional group for the purposes of biosensing, ${ }^{8}$ and multiple cages/biosensors could be simultaneously employed to allow multiplexed bioanalyte detection. The key feature empowering the biosensor approach is that the chemical shift of Xe within a biosensor cage that has bound a bioanalyte is measurably different from that of Xe residing within an unbound cage — thereby allowing the binding event to be detected via HP ${ }^{129} \mathrm{Xe}$ MRS. It is expected that for in vivo applications, rather than delivering Xe-loaded biosensors to the body in a single bolus, one would first administer the biosensors to the subject and allow them time to bind to their biomolecular targets. Then later, HP Xe may be delivered (using an above method) so that it would have the opportunity to locate the biosensor cages immediately prior to MRS.

$<$ Figure 10 near here $>$

This type of direct-detection experiment would yield three types of ${ }^{129}$ Xe peaks: one for unbound $\mathrm{Xe}$ in the bulk environment, one for Xe trapped in an unbound host biosensor molecule, and one for Xe residing in biosensor molecules bound to targeted analytes. However, if the targeted bioanalyte is in low concentration, the bound Xe signal can be buried in noise. One way around this problem is to employ HP chemical exchange saturation 
transfer, or Hyper-CEST. ${ }^{59}$ Hyper-CEST requires that the exchange of HP nuclei between sites is slow enough to give rise to unique, addressable resonances-yet fast enough to allow significant fractions of the populations to undergo exchange during the experiment. ${ }^{8}$ Thus, if a saturating RF pulse is applied at the frequency for a bound-Xe resonance, it will reduce the magnetization not only for the bound Xe pool, but also for the (typically much larger) bulk Xe pool as well: off-resonance saturation provides a control/reference signal. The greater sensitivity afforded by indirectly measuring the presence of activated biosensors via the more sensitive bulk-Xe signal allows detection of concentrations as low as $\sim 100 \mathrm{nM}$, with further optimization not inconceivably improving the detection limit to the $\sim 700 \mathrm{fM}$ range. ${ }^{8}$

\section{CONCLUSIONS}

In summary, four hyperpolarization techniques have been described here in detail that either successfully enable in vivo MRS studies (d-DNP, PHIP, and SEOP/Xe), or have a strong potential for in vivo MRS in the near future (SABRE). Key features of these hyperpolarization methods from the perspective of their biomedical use are compared in Table 1. Some of these techniques have evolved rapidly from proof-of-principle studies using custom-built instrumentation to robust commercial equipment. This in turn has fueled reliable in vivo experimentation - including the first clinical trials in human volunteers. The ongoing innovations in instrumentation and the fundamental technology of hyperpolarization will likely continue to significantly and positively impact access and applications for the broader biomedical community, expanding the utility of HP methods as a new tool for probing fundamental biomedical questions. 
Table 1. Comparison of four hyperpolarization methods.

\begin{tabular}{|c|c|c|c|c|}
\hline Parameter & d-DNP & PHIP & SABRE & SEOP/Xe \\
\hline Wide range of agents & yes & no & no & no \\
\hline Straightforward scalability & no & yes & yes & yes \\
\hline $\begin{array}{l}\text { High }(>50 \% \text { / order-unity }) \\
\text { polarization }\end{array}$ & yes & yes & no & yes \\
\hline $\begin{array}{l}\text { Moderately high }(>1 \%) \\
\text { polarization }\end{array}$ & yes & yes & yes & yes \\
\hline Continuous generation & no & yes & yes & yes \\
\hline $\begin{array}{l}\text { Different modes of } \\
\text { production, i.e. continuous } \\
\text { (C) vs. batch (B)/stopped- } \\
\text { flow (SF)) }\end{array}$ & $\mathrm{B} / \mathrm{SF}$ & $\mathrm{C} \& \mathrm{~B} / \mathrm{SF}$ & $\mathrm{C} \& \mathrm{~B} / \mathrm{SF}$ & $\mathrm{C} \& \mathrm{~B} / \mathrm{SF}$ \\
\hline Preparation of 'pure' agents & yes & $\begin{array}{l}\text { under } \\
\text { development }\end{array}$ & $\begin{array}{l}\text { under } \\
\text { development }\end{array}$ & yes \\
\hline $\begin{array}{l}\text { Preparation of concentrated } \\
\text { agent suitable for in vivo } \\
\text { MRS use }\end{array}$ & yes & yes & no & yes \\
\hline $\begin{array}{l}\text { Long-lived in vitro agent } \\
\text { (relaxation time }>1 \mathrm{~min} \text { ) }\end{array}$ & yes & yes & no & yes \\
\hline $\begin{array}{l}\text { Long-lived in vivo agent } \\
\text { (relaxation time }>0.5 \mathrm{~min} \text { ) }\end{array}$ & yes & yes & no & no \\
\hline Technology Cost & high & low & low & medium \\
\hline Operational Cost & high & low & low & medium \\
\hline $\begin{array}{l}\text { Commercially available } \\
\text { device }\end{array}$ & yes/multiple & $\begin{array}{l}\text { no (but yes } \\
\text { for para- } \mathrm{H}_{2} \\
\text { generator) }\end{array}$ & yes & yes \\
\hline Phase of agent & gas/liq/solid & liq/gas & liq & gas \\
\hline $\begin{array}{l}\text { Environment Sensing (ES) } \\
\text { and penetration of } \\
\text { biochemical pathways (BP) }\end{array}$ & $\mathrm{BP}$ & BP \& ES & - & ES \\
\hline
\end{tabular}




\section{RERERENCES}

1. J. H. Ardenkjaer-Larsen, B. Fridlund, A. Gram, G. Hansson, L. Hansson, M. H. Lerche, R. Servin, M. Thaning and K. Golman, Proc. Natl. Acad. Sci. U. S. A., 2003, 100, 10158.

2. J. Kurhanewicz, D. B. Vigneron, K. Brindle, E. Y. Chekmenev, A. Comment, C. H. Cunningham, R. J. DeBerardinis, G. G. Green, M. O. Leach, S. S. Rajan, R. R. Rizi, B. D. Ross, W. S. Warren and C. R. Malloy, Neoplasia, 2011, 13, 81.

3. R. Sriram, J. Kurhanewicz and D. B. Vigneron. (2014). emrstm1253. In eMagRes, pp. 311: John Wiley \& Sons, Ltd.

4. S. J. Nelson, J. Kurhanewicz, D. B. Vigneron, P. E. Z. Larson, A. L. Harzstark, M. Ferrone, M. van Criekinge, J. W. Chang, R. Bok, I. Park, G. Reed, L. Carvajal, E. J. Small, P. Munster, V. K. Weinberg, J. H. Ardenkjaer-Larsen, A. P. Chen, R. E. Hurd, L. I. Odegardstuen, F. J. Robb, J. Tropp and J. A. Murray, Sci. Transl. Med., 2013, 5, 198ra108.

5. R. A. Green, R. W. Adams, S. B. Duckett, R. E. Mewis, D. C. Williamson and G. G. R. Green, Prog. Nucl. Mag. Res. Sp., 2012, 67, 1.

6. P. Nikolaou, B. M. Goodson and E. Y. Chekmenev, Chem. Eur. J., 2015, 21, 3156.

7. B. M. Goodson, J. Magn. Reson., 2002, 155, 157.

8. L. Schroder, Phys. Medica, 2013, 29, 3.

9. A. Comment and M. E. Merritt, Biochemistry, 2014, 53, 7333.

10. R. G. Griffin and T. F. Prisner, Phys. Chem. Chem. Phys., 2010, 12, 5737.

11. T. Maly, G. T. Debelouchina, V. S. Baja, K.-N. Hu, C.-G. Joo, M. L. Mak-Jurkauskas, J. R. Sirigiri, P. C. A. van der Wel, J. Herzfeld, R. J. Temkin and R. G. Griffin, J. Chem. Phys., 2008, 128, 052211.

12. J. H. Ardenkjaer-Larsen, A. M. Leach, N. Clarke, J. Urbahn, D. Anderson and T. W. Skloss, NMR Biomed., 2011, 24, 927.

13. P. Dutta, G. Martinez and R. Gillies, Biophys. Rev., 2013, 5, 271. 
14. H. Johannesson, S. Macholl and J. H. Ardenkjaer-Larsen, J. Magn. Reson., 2009, 197, 167.

15. Y. Lee, N. M. Zacharias, D. Piwnica-Worms and P. K. Bhattacharya, Chem. Comm., 2014, 50, 13030.

16. K.-N. Hu, H.-H. Yu, T. M. Swager and R. G. Griffin, J. Am. Chem. Soc., 2004, 126, 10844

17. T. R. Eichhorn, Y. Takado, N. Salameh, A. Capozzi, T. Cheng, J.-N. Hyacinthe, M.

Mishkovsky, C. Roussel and A. Comment, Proc. Natl. Acad. Sci. U. S. A., 2013, 110, 18064.

18. L. Lumata, Z. Kovacs, C. Malloy, A. D. Sherry and M. Merritt, Phys. Med. Biol., 2011, 56, N85.

19. A. Farkas, 'Ortho-hydrogen, para-Hydrogen, and Heavy Hydrogen', Cambridge University Press, Cambridge, UK, 1935.

20. L. Pauling and E. B. Wilson Jr, 'Introduction to quantum mechanics, with applications to chemistry, New edition.', New edition., Dover, Mineola, New York, United States, 1935.

21. J. B. Hövener, S. Bar, J. Leupold, K. Jenne, D. Leibfritz, J. Hennig, S. B. Duckett and D. von Elverfeldt, NMR Biomed., 2013, 26, 124.

22. S. Kadlecek, V. Vahdat, T. Nakayama, D. Ng, K. Emami and R. Rizi, NMR Biomed., 2011, 24, 933.

23. B. Feng, A. M. Coffey, R. D. Colon, E. Y. Chekmenev and K. W. Waddell, J. Magn. Reson., 2012, 214, 258.

24. C. R. Bowers and D. P. Weitekamp, Phys. Rev. Lett., 1986, 57, 2645.

25. T. C. Eisenschmid, R. U. Kirss, P. P. Deutsch, S. I. Hommeltoft, R. Eisenberg, J. Bargon, R. G. Lawler and A. L. Balch, J. Am. Chem. Soc., 1987, 109, 8089.

26. K. Golman, O. Axelsson, H. Johannesson, S. Mansson, C. Olofsson and J. S. Petersson, Magn. Reson. Med., 2001, 46, 1. 
27. M. Goldman and H. Johannesson, C. R. Physique, 2005, 6, 575.

28. K. V. Kovtunov, M. L. Truong, D. L. Barskiy, O. G. Salnikov, V. I. Bukhtiyarov, A. M. Coffey, K. W. Waddell, I. V. Koptyug and E. Y. Chekmenev, J. Phys. Chem. C, 2014, 118, 28234.

29. R. V. Shchepin, A. M. Coffey, K. W. Waddell and E. Y. Chekmenev, Anal. Chem., 2014, 86, 5601 .

30. N. M. Zacharias, H. R. Chan, N. Sailasuta, B. D. Ross and P. Bhattacharya, J. Am. Chem. Soc., 2012, 134, 934.

31. P. Bhattacharya, E. Y. Chekmenev, W. F. Reynolds, S. Wagner, N. Zacharias, H. R.

Chan, R. Bünger and B. D. Ross, NMR Biomed., 2011, 24, 1023.

32. K. V. Kovtunov, V. V. Zhivonitko, I. V. Skovpin, D. A. Barskiy and I. V. Koptyug, Top. Curr. Chem., 2013, 338, 123.

33. M. Irfan, N. Eshuis, P. Spannring, M. Tessari, M. C. Feiters and F. P. J. T. Rutjes, J. Phys. Chem. C, 2014, 118, 13313.

34. L. S. Bouchard, S. R. Burt, M. S. Anwar, K. V. Kovtunov, I. V. Koptyug and A. Pines, Science, 2008, 319, 442.

35. J.-B. Hövener, E. Chekmenev, K. Harris, W. Perman, L. Robertson, B. Ross and P. Bhattacharya, Magn. Reson. Mater. Phy., 2009, 22, 111.

36. K. W. Waddell, A. M. Coffey and E. Y. Chekmenev, J. Am. Chem. Soc., 2011, 133, 97.

37. R. W. Adams, J. A. Aguilar, K. D. Atkinson, M. J. Cowley, P. I. P. Elliott, S. B. Duckett, G. G. R. Green, I. G. Khazal, J. Lopez-Serrano and D. C. Williamson, Science, 2009, 323, 1708.

38. M. J. Cowley, R. W. Adams, K. D. Atkinson, M. C. R. Cockett, S. B. Duckett, G. G. R. Green, J. A. B. Lohman, R. Kerssebaum, D. Kilgour and R. E. Mewis, J. Am. Chem. Soc., 2011, 133, 6134. 
39. H. Zeng, J. Xu, J. Gillen, M. T. McMahon, D. Artemov, J.-M. Tyburn, J. A. B. Lohman, R. E. Mewis, K. D. Atkinson, G. G. R. Green, S. B. Duckett and P. C. M. van Zijl, J. Magn. Reson., 2013, 237, 73.

40. T. Theis, M. L. Truong, A. M. Coffey, R. V. Shchepin, K. W. Waddell, F. Shi, B. M. Goodson, W. S. Warren and E. Y. Chekmenev, J. Am. Chem. Soc., 2015, 137, 1404.

41. D. A. Barskiy, K. V. Kovtunov, I. V. Koptyug, P. He, K. A. Groome, Q. A. Best, F. Shi, B. M. Goodson, R. V. Shchepin, A. M. Coffey, K. W. Waddell and E. Y. Chekmenev, J. Am. Chem. Soc., 2014, 136, 3322.

42. M. L. Truong, F. Shi, P. He, B. Yuan, K. N. Plunkett, A. M. Coffey, R. V. Shchepin, D. A. Barskiy, K. V. Kovtunov, I. V. Koptyug, K. W. Waddell, B. M. Goodson and E. Y. Chekmenev, J. Phys. Chem. B, 2014, 1813882.

43. A. N. Pravdivtsev, A. V. Yurkovskaya, H.-M. Vieth and K. L. Ivanov, Phys. Chem. Chem. Phys., 2014, 16, 24672.

44. S. Gloggler, R. Muller, J. Colell, M. Emondts, M. Dabrowski, B. Blumich and S. Appelt, Phys. Chem. Chem. Phys., 2011, 13, 13759.

45. F. Shi, A. M. Coffey, K. W. Waddell, E. Y. Chekmenev and B. M. Goodson, Angew. Chem. Int. Ed., 2014, 53, 7495.

46. T. G. Walker and W. Happer, Rev. Mod. Phys., 1997, 69, 629.

47. M. E. Wagshul and T. E. Chupp, Phys. Rev. A, 1989, 40, 4447.

48. P. Nikolaou, A. M. Coffey, L. L. Walkup, B. M. Gust, N. Whiting, H. Newton, S. Barcus, I. Muradyan, M. Dabaghyan, G. D. Moroz, M. Rosen, S. Patz, M. J. Barlow, E. Y. Chekmenev and B. M. Goodson, Proc. Natl. Acad. Sci. U. S. A., 2013, 110, 14150.

49. B. Driehuys, G. D. Cates, E. Miron, K. Sauer, D. K. Walter and W. Happer, Appl. Phys. Lett., 1996, 69, 1668.

50. A. L. Zook, B. B. Adhyaru and C. R. Bowers, J. Magn. Reson., 2002, 159, 175. 
51. I. C. Ruset, S. Ketel and F. W. Hersman, Phys. Rev. Lett., 2006, 96, 053002.

52. N. N. Kuzma, B. Patton, K. Raman and W. Happer, Phys. Rev. Lett., 2002, 88, 147602.

53. M. S. Rosen, T. E. Chupp, K. P. Coulter, R. C. Welsh and S. D. Swanson, Rev. Sci. Instrum., 1999, 70, 1546.

54. P. Nikolaou, A. M. Coffey, M. J. Barlow, M. Rosen, B. M. Goodson and E. Y. Chekmenev, Anal. Chem., 2014, 86, 8206.

55. E. Babcock, I. Nelson, S. Kadlecek, B. Driehuys, L. W. Anderson, F. W. Hersman and T. G. Walker, Phys. Rev. Lett., 2003, 91, 4.

56. Z. I. Cleveland, H. E. Moller, L. W. Hedlund and B. Driehuys, J. Phys. Chem. B, 2009, 113, 12489.

57. J. Wolber, A. Cherubini, M. O. Leach and A. Bifone, Magn. Reson. Med., 2000, 43, 491. 58. M. M. Spence, S. M. Rubin, I. E. Dimitrov, E. J. Ruiz, D. E. Wemmer, A. Pines, S. Q. Yao, F. Tian and P. G. Schultz, Proc. Natl. Acad. Sci. U. S. A., 2001, 98, 10654.

59. L. Schroder, T. J. Lowery, C. Hilty, D. E. Wemmer and A. Pines, Science, 2006, 314, 446. 


\section{Figure Captions}

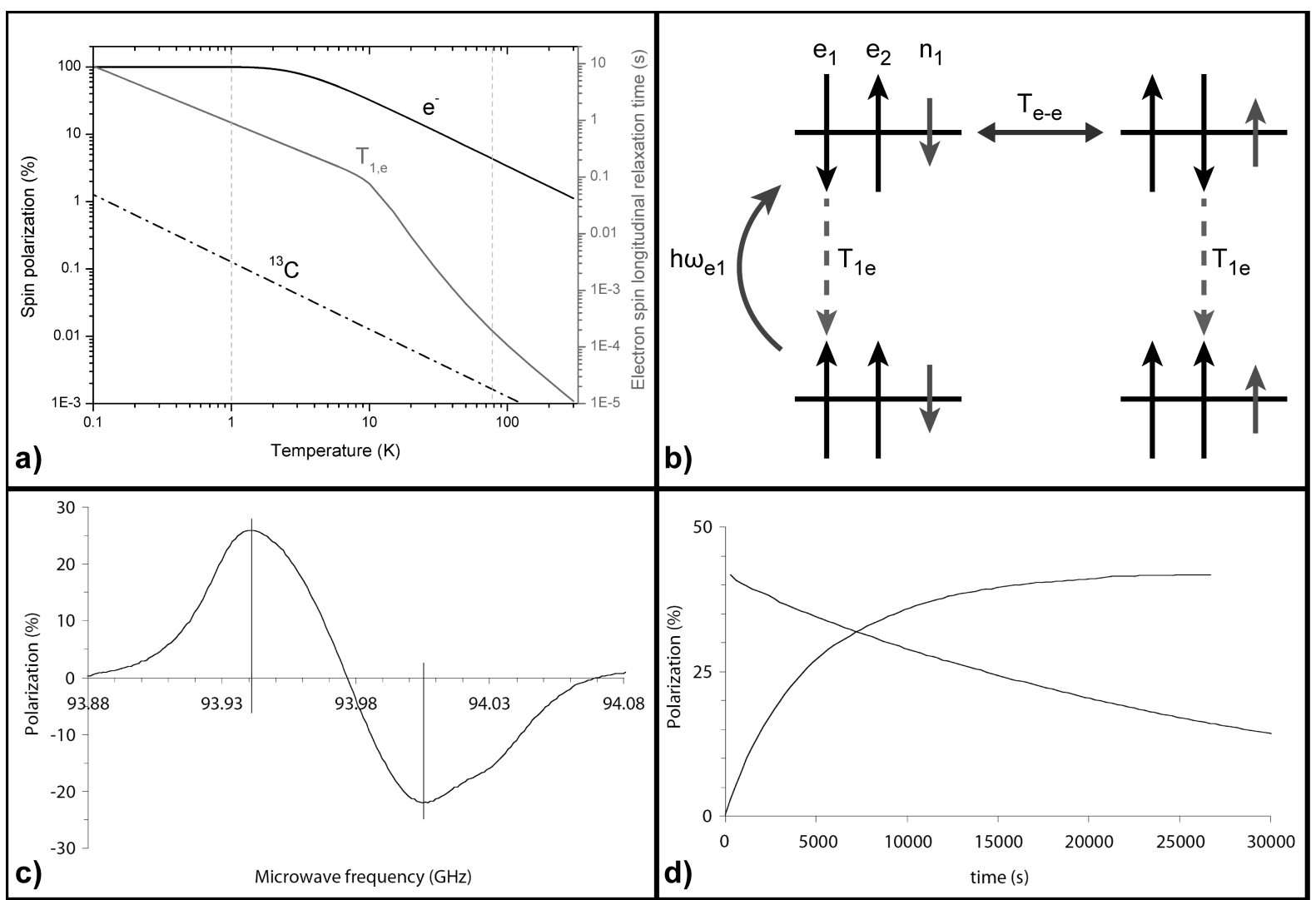

Figure 1. Effects of DNP conditions on electronic and nuclear spins. (a). Effects of temperature on ${ }^{13} \mathrm{C}$ (dashed black curve) and electron ( $\mathrm{e}^{-}$, solid black curve) spin polarizations at $5 \mathrm{~T}$, along with electronic $T_{1}$ relaxation time $\left(\mathrm{T}_{1, \mathrm{e}}\right.$, gray solid curve) at $0.35 \mathrm{~T}$. The figure is reprinted with permission from A. Comment and M. E. Merritt, Biochemistry, 2014, 53, 7333. ${ }^{9}$ Copyright 2014 American Chemical Society. (b) The response of a twoelectron nuclear spin system during the DNP process: microwave irradiation $\left(h \omega_{\mathrm{e} 1}\right)$ induces spin-flips of coupled electrons (left), which then cross-relax with nearby nuclei (top right), driving the nuclear spins into the same HP spin state (bottom right). (c) Example of a microwave sweep used to calibrate the optimal excitation frequency for ${ }^{13} \mathrm{C}$ DNP. (d) Examplary buildup and decay of ${ }^{13} \mathrm{C}$ polarization over time with the DNP microwave source engaged (increasing curve) and turned-off (decreasing curve). Parts (c) \& (d) are from Ardenkjaer-Larsen, et al. PNAS 100.18 (2003) 10158-10163; Copyright (2003) National Academy of Sciences, U.S.A. ${ }^{1}$ 


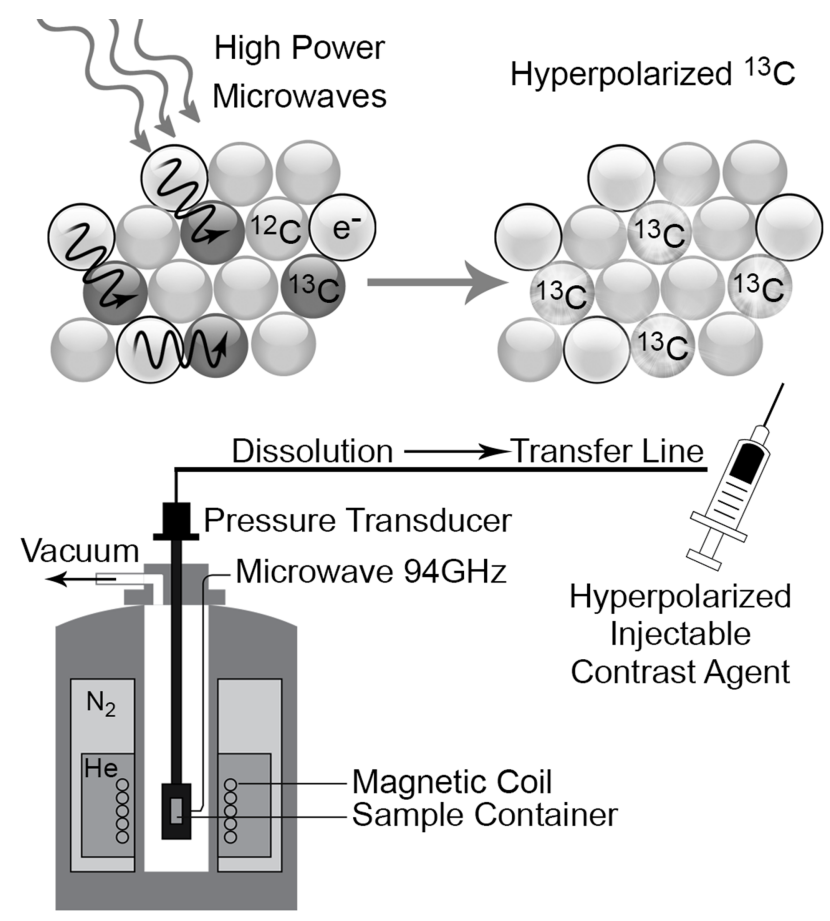

Figure 2. Overview of the d-DNP process. Within the high magnetic field of the DNP polarizer, high-power microwaves excite free electrons in free radical species mixed with the HP substrate and frozen into an amorphous solid. The electrons undergo spin-exchange with nearby ${ }^{13} \mathrm{C}$ nuclei and over time, the entire sample becomes polarized via direct dipole-dipole interactions as well as nuclear spin diffusion involving the ${ }^{1} \mathrm{H}$ nuclei present in the HP substrate or glassing agent. After polarization, the HCA sample is rapidly dissolved in hot solvent and shuttled into the MR scanner room, for injection into the animal or human subject. 
a)

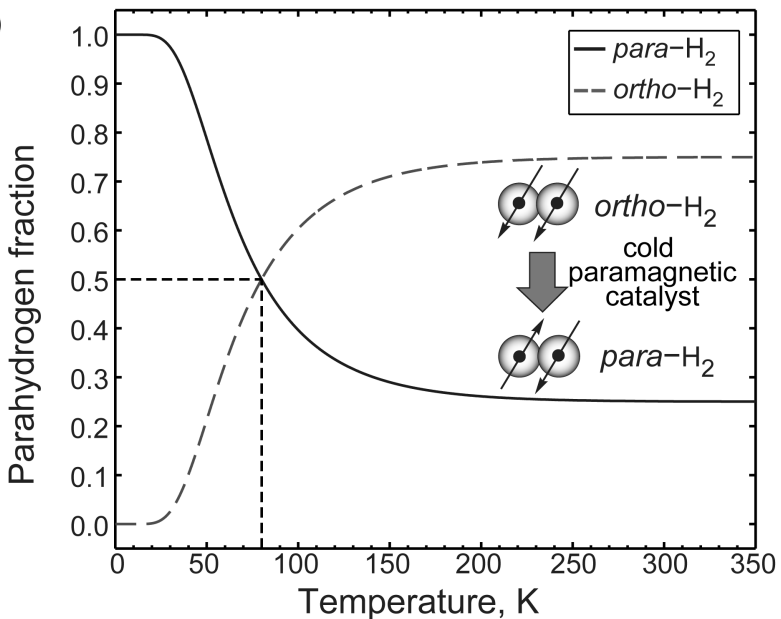

b)

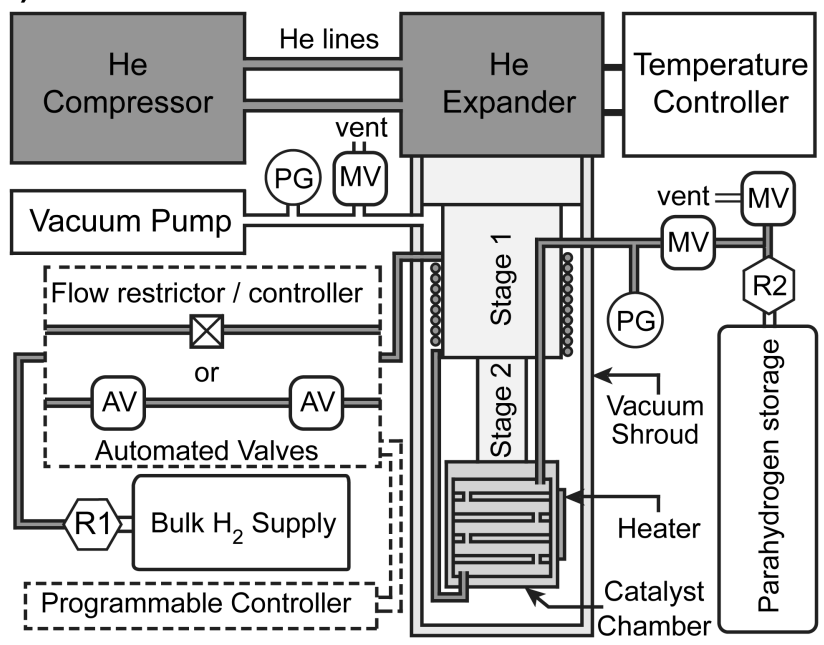

Figure 3. Production of para-hydrogen (para- $\left.\mathrm{H}_{2}\right)$. (a) $\mathrm{H}_{2}$ passes over a paramagnetic

catalyst which rapidly increases the para- $\mathrm{H}_{2}$ fraction to the thermodynamic equilibrium at the given cryogenic temperature. (b) Production typically involves a "parahydrogen generator" built around a two-stage He cryocooled cold-head involving pressure gauges (PG), manual valves (MV), gas pressure regulators (R), and automated valves (AV). 


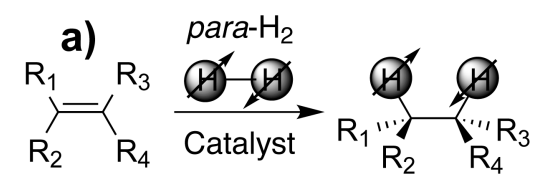

b)

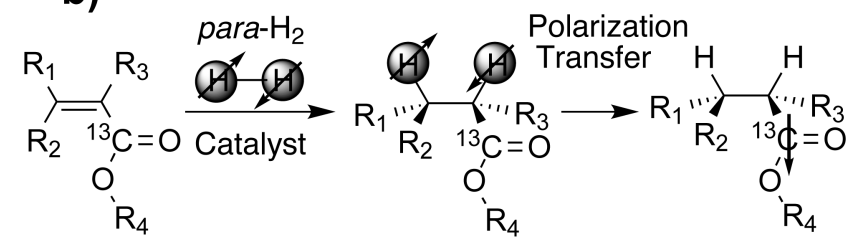

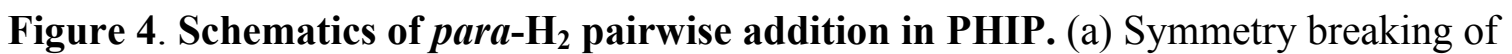
nascent para-hydrogen's nuclear spin state via chemical reaction creates a non-symmetric product. (b) para- $\mathrm{H}_{2}$ state symmetry-breaking through chemical reaction with pairwise addition followed by polarization transfer to ${ }^{13} \mathrm{C}$, produces a HP contrast agent comprised of a ${ }^{13} \mathrm{C}$ HP carboxyl moiety with a long $T_{1}$ lifetime. 


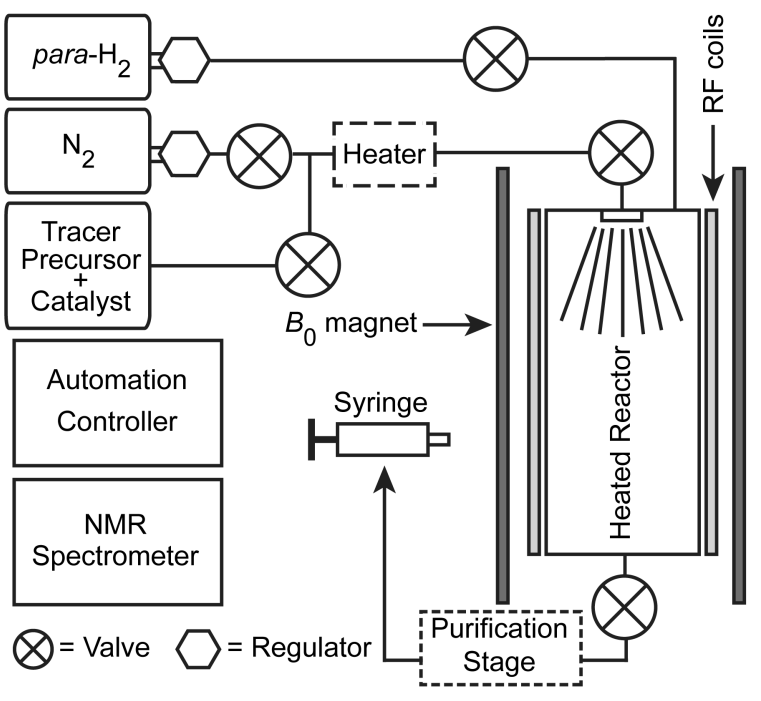

Figure 5. Generic PHIP hyperpolarizer schematic. Optional elements are boxes with dotted lines. In the PHIP hyperpolarization cycle: (i) para- $\mathrm{H}_{2}$ is filled in the previously deoxygenated (via extensive $\mathrm{N}_{2}$ gas venting) heated reactor, (ii) "tracer PHIP precursor and catalyst" solution is loaded into an optional "Heater" element followed by (iii) rapid solution spraying in the para- $\mathrm{H}_{2}$-filled reactor under conditions of ${ }^{1} \mathrm{H}$ decoupling; (iv) when the spraying is finished (a few seconds), the polarization transfer sequence immediately transforms ( $<1$ second) hyperpolarization from nascent parahydrogen protons (see Figure 4b) to hyperpolarization on the ${ }^{13} \mathrm{C}$ nucleus; (v) the solution containing the ${ }^{13} \mathrm{C}$ HP compound is ejected from the reactor through an optional purification stage for in vivo administration. The system can be purged with $\mathrm{N}_{2}$ gas (typically $\sim 1 \mathrm{~min}$ ) to remove any residual solution from the PTFE lines to prepare for the next PHIP hyperpolarization production cycle. 


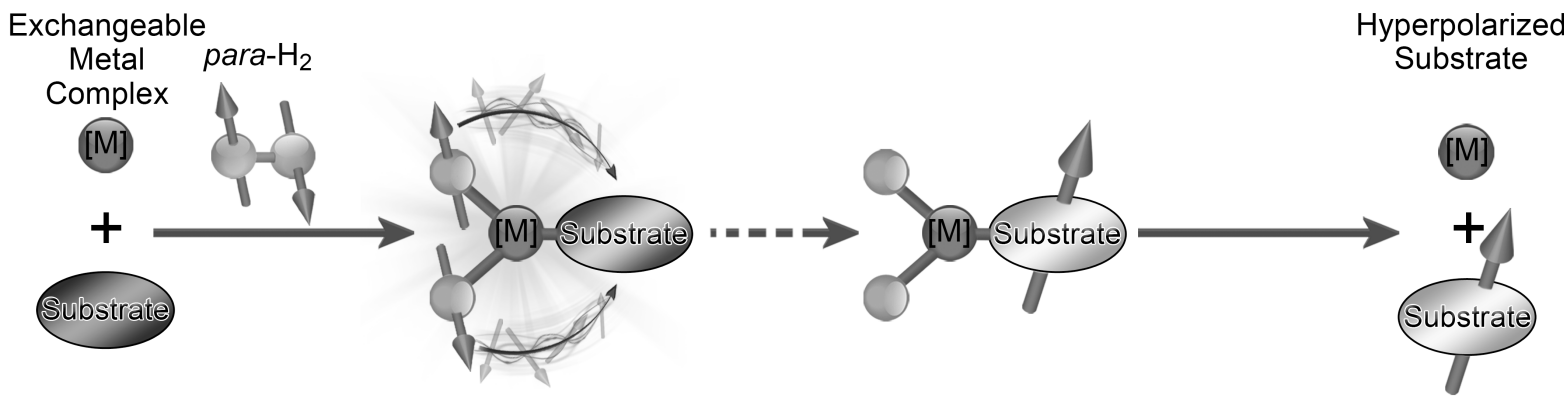

Figure 6. Schematic of the SABRE process. Reversible exchange of para- $\mathrm{H}_{2}$ and the substrate on a metal catalyst ([M]) leads to a HP substrate. The figure is adapted from Ref. ${ }^{6}$.

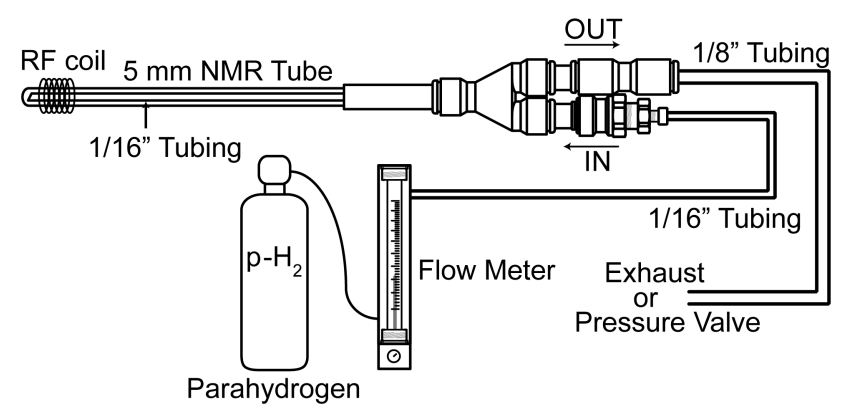

Figure 7. Experimental setup for SABRE. Para- $\mathrm{H}_{2}$ bubbles through a catalytic solution in a conventional $5 \mathrm{~mm}$ NMR tube. The solution sits in a medium-wall-thickness NMR tube where para $-\mathrm{H}_{2}$ is delivered via Teflon ${ }^{\circledR}$ tubing, and the expended gas leaves via an exhaust line that can be capped with a pressure-calibrated safety valve to allow SABRE at elevated para $-\mathrm{H}_{2}$ pressure. The figure is reprinted with permission from M. L. Truong, et al., J. Phys. Chem. B, 2014, 18, 13882. ${ }^{42}$ Copyright 2014 American Chemical Society. 


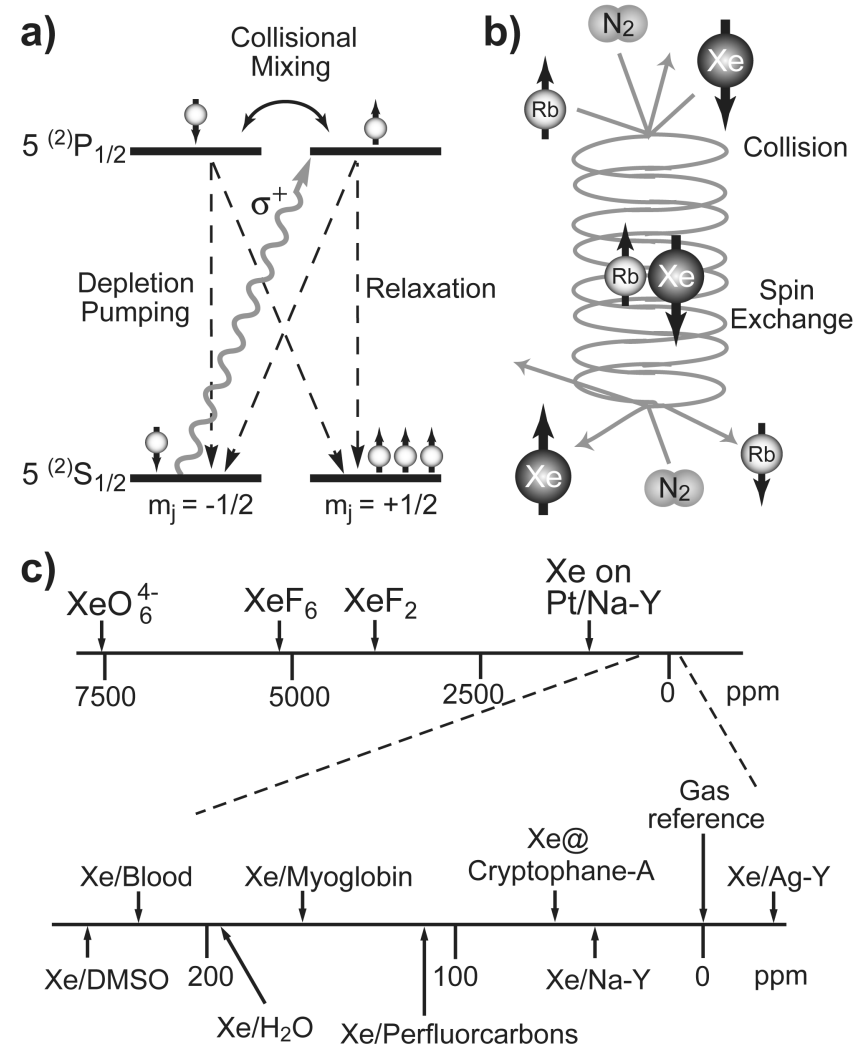

Figure 8. SEOP hyperpolarization and chemical-shift sensitivity of ${ }^{129}$ Xe. (a) Application of circularly polarized laser light and subsequent non-radiative relaxation results in depletion of one $\mathrm{Rb}$ electron $\left(m_{\mathrm{j}}\right)$ level and accrual of population in the other, rendering the $\mathrm{Rb}$ electronically spin-polarized (Rb nuclear spin levels not shown). (b) Gas-phase collisions allow spin-exchange between $\mathrm{Rb}$ electrons and ${ }^{129} \mathrm{Xe}$ nuclei, resulting in $\mathrm{HP}{ }^{129} \mathrm{Xe}$ with time. A three-body collision is shown in this example, with $\mathrm{N}_{2}$ molecules facilitating formation and break-up of the complex. (c) Chemical shifts for ${ }^{129} \mathrm{Xe}$ in different environments. Figures are reprinted from Nuclear magnetic resonance of laser-polarized noble gases in molecules, materials, and organisms. B. M. Goodson, J. Magn. Reson. 2002, 155, 157, Copyright (2002), ${ }^{7}$ with permission from Elsevier. 

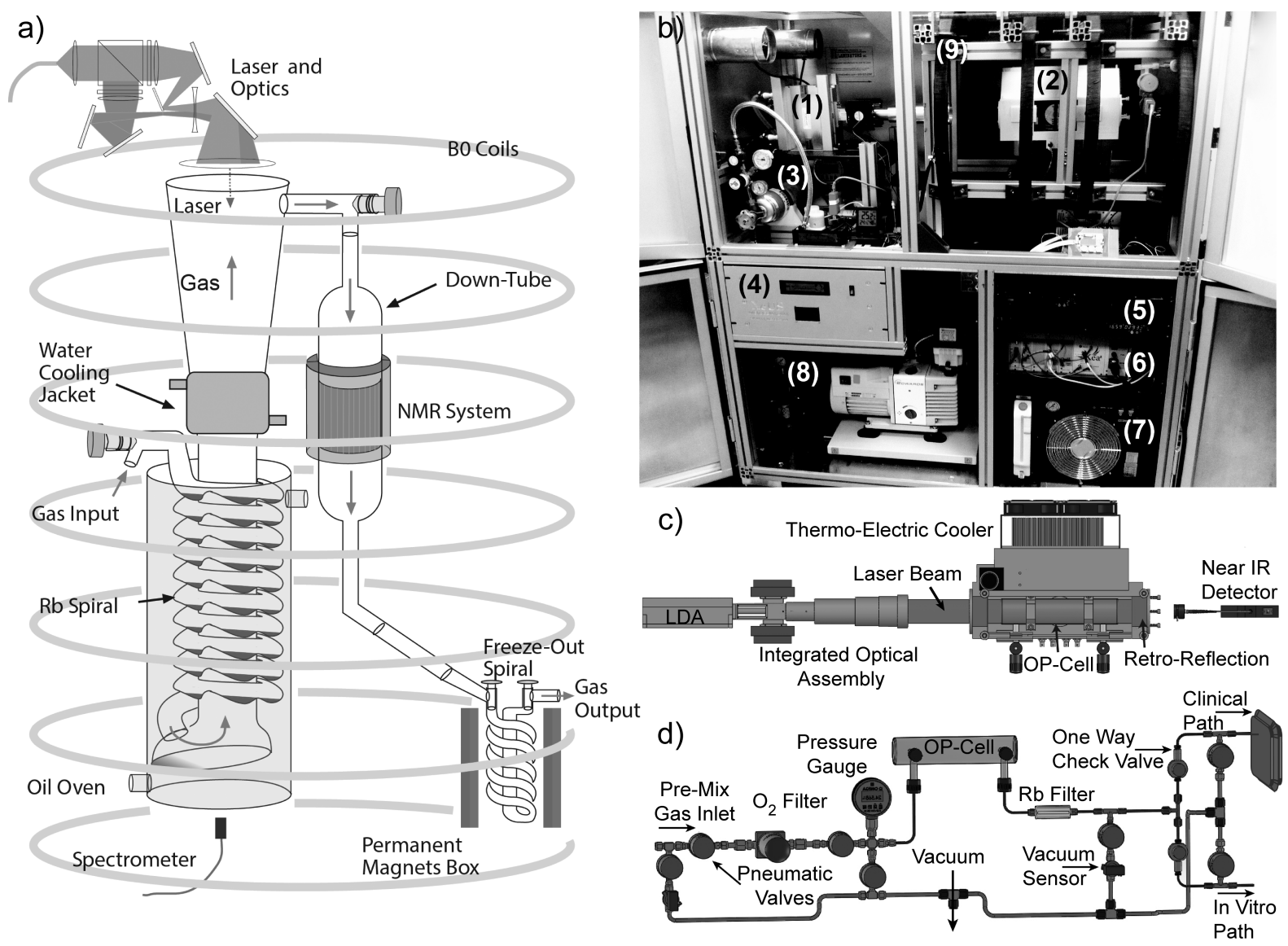

Figure 9. Examples of ${ }^{129}$ Xe hyperpolarizers. (a). Diagram showing the CF design of Ruset et al. ${ }^{51}$. Figure courtesy of W. Hersman, reprinted with permission from I. C. Ruset, S. Ketel, F. W. Hersman, Phys. Rev. Lett. 2006, 96, 053002. Copyright (2006) by the American Physical Society. (b-d). Our Consortium's second-generation SF Xe hyperpolarizer. The photo in (b) shows the principal components of the device [(1) laser; (2) 3D-printed oven; (3) Xe gas supply; (4) microcontroller box; (5) power supplies; (6) low-frequency NMR spectrometer; (7) water chiller; (8) vacuum pumps; (9) electromagnetic coils]. Diagrams showing the oven/optical path and gas manifold are shown respectively in (c) and (d). Figure $\mathrm{d}$ is reprinted with permission from P. Nikolaou, et al., Anal. Chem., 2014, 86, $8206 .{ }^{54}$ Copyright 2014 American Chemical Society. 

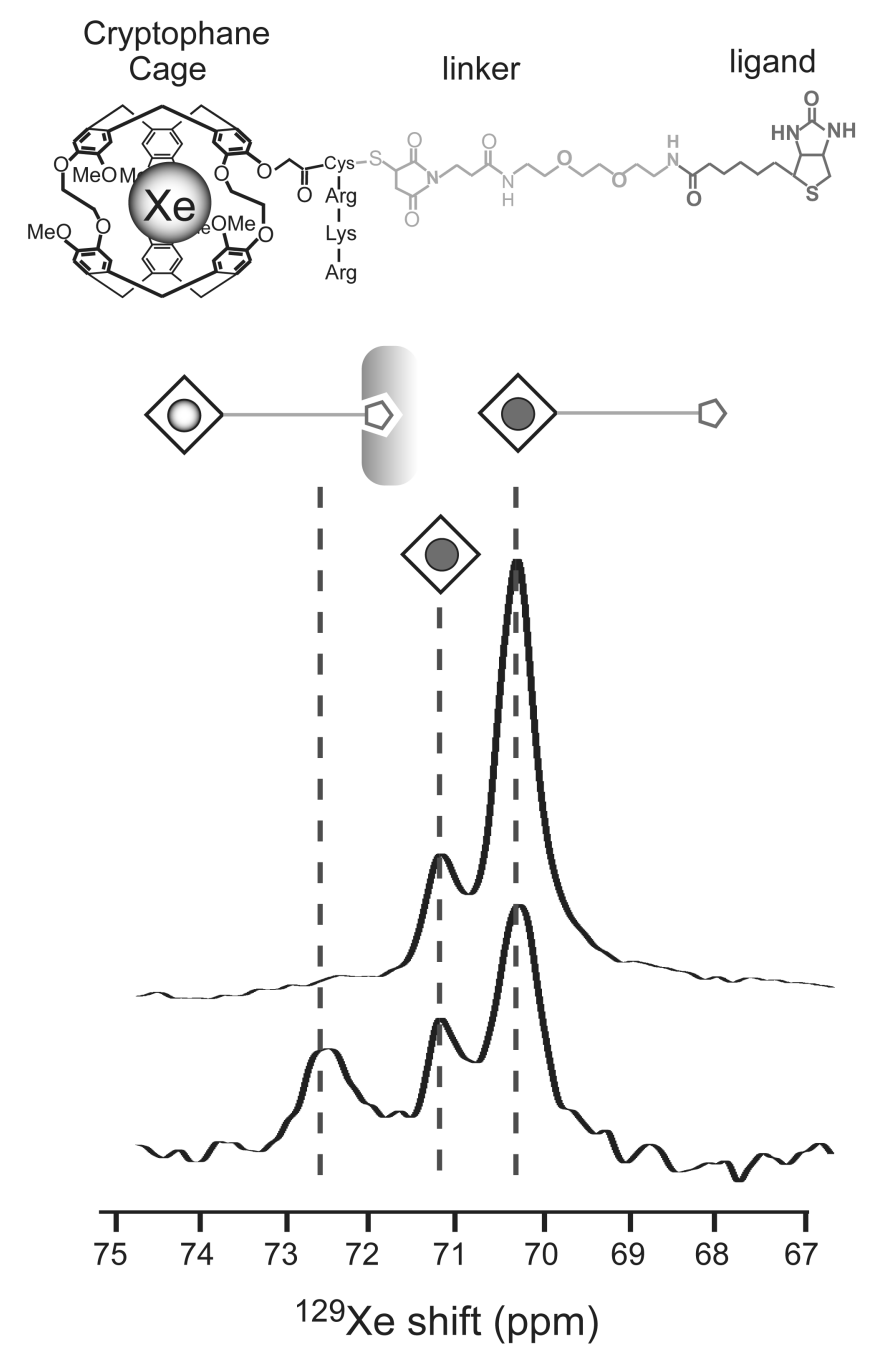

Figure 10. Structure and MR of a xenon biosensor. The chemical structure of a xenon biosensor molecule comprises a cryptophane cage, a linker group, and a molecule (e.g. biotin) to bind to a targeted bioanalyte (e.g. avidin) with high affinity and specificity. ${ }^{58}$ Below the structure are ${ }^{129} \mathrm{Xe}$ spectra monitoring the binding of biotin-functionalized xenon to the prototypical bioanalyte avidin. The spectra show only the cryptophane-bound peaks; the (much larger) peak corresponding to free xenon in water is at $193 \mathrm{ppm}$. The spectra differ by the absence (top) and presence (bottom) of $80 \mathrm{nmol}$ avidin in solution, manifesting in the bottom spectrum by the appearance of a new peak at $~ 72.5 \mathrm{ppm}$. Adapted from figures courtesy of Prof. Alex Pines. 